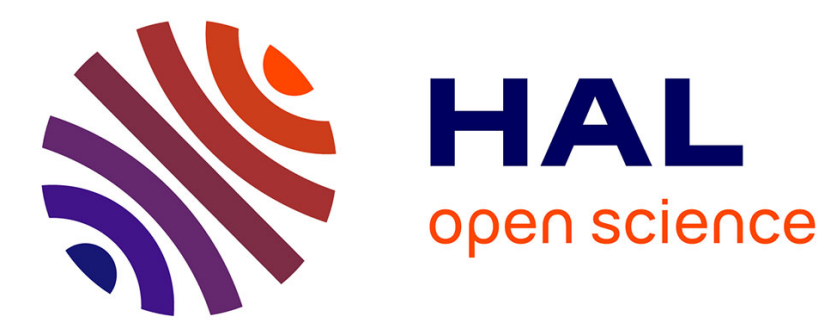

\title{
On a numerical shape optimization approach for a class of free boundary problems
}

\author{
A. Boulkhemair, A. Chakib, A. Nachaoui, A A Niftiyev, A Sadik
}

\section{To cite this version:}

A. Boulkhemair, A. Chakib, A. Nachaoui, A A Niftiyev, A Sadik. On a numerical shape optimization approach for a class of free boundary problems. 2019. hal-02073376v2

\section{HAL Id: hal-02073376 \\ https://hal.science/hal-02073376v2}

Preprint submitted on 8 Jul 2019

HAL is a multi-disciplinary open access archive for the deposit and dissemination of scientific research documents, whether they are published or not. The documents may come from teaching and research institutions in France or abroad, or from public or private research centers.
L'archive ouverte pluridisciplinaire HAL, est destinée au dépôt et à la diffusion de documents scientifiques de niveau recherche, publiés ou non, émanant des établissements d'enseignement et de recherche français ou étrangers, des laboratoires publics ou privés. 


\title{
On a numerical shape optimization approach for a class of free boundary problems
}

\author{
A. Boulkhemair ${ }^{\mathrm{a}, 1}$, A. Chakib ${ }^{\mathrm{b}, 2}$, A. Nachaoui ${ }^{\mathrm{a}, 1}$, A.A.Niftiyev ${ }^{\mathrm{c}}$ and A. Sadik ${ }^{\mathrm{a}, \mathrm{b}}$ \\ a'Laboratoire de Mathématiques Jean Leray UMR6629 CNRS, \\ UFR - Sciences et Techniques Nantes, \\ 2 rue de la Houssinière, BP92208 44322 Nantes, France. \\ ${ }^{1}$ E-mail : boulkhemair-a@univ-nantes.fr \& Abdeljalil.Nachaoui@univ-nantes.fr \\ ${ }^{b}$ Laboratoire de Mathématiques et Applications, \\ Université Sultan Moulay Slimane, Faculté des Sciences et Techniques, \\ B.P.523, Béni-Mellal, Maroc. \\ ${ }^{2}$ E-mail : chakib.fstbm@gmail.com \\ ${ }^{\mathrm{c}}$ Institute of Applied Mathematics, Baku state University, Azerbaijan.
}

Dedicated to the memory of Professor Agaddin A. Niftiyev.

July 8th, 2019

\begin{abstract}
This paper is devoted to a numerical method for the approximation of a class of free boundary problems of Bernoulli's type, reformulated as optimal shape design problems with appropriate shape functionals. We show the existence of the shape derivative of the cost functional on a class of admissible domains and compute its shape derivative by using the formula proposed in [6,7], that is, by means of support functions. On the numerical level, this allows us to avoid the tedious computations of the method based on vector fields. A gradient method combined with boundary element method are performed for the approximation of this problem, in order to overcome the re-meshing task required by the finite element method. Finally, we present some numerical results and simulations concerning practical applications, showing the effectiveness of the proposed approach.
\end{abstract}

Keywords shape optimization, free boundary problem, Bernoulli problem, optimal solution, shape derivative, convex domain, support function, cost functional.

\section{Introduction}

The main target of shape optimization is to provide a common and systematic framework for optimizing structures described by various practical physical or mechanical models; especially, hydrodynamics, elasticity, geophysics 
and aerodynamics models. Shape optimization problems consist in finding the optimal shape (or domain) which minimizes a certain cost functional under given constraints such as a partial differential equation defined on the variable domain. Since the seventies of the last century, many authors investigated the shape optimization field and remarkable progress has been achieved in shape and topology optimization. In fact, the growing interest in this field reflects a growing sophistication in structural analysis and optimization which allow solving more and more difficult shape optimization problems. However, one may say that no uniform approach to shape optimization problems has yet emerged.

The numerical investigation of shape optimization problems is based on the study of the first variation of the cost functional, and in particular on the computation of its gradient. So, as the variation of the domain is characterized by the variation of its boundary, in this process arise both numerical and theoretical difficulties. The method of variation of domains using vector fields, introduced by Céa [12] and developed then, among others, by Murat, Simon, Sokolowski, Delfour and Zolésio [13,26], Allaire [2] and Henrot and Pierre [19], allowed to solve some of these issues. But these techniques themselves present some difficulties from both theoretical and numerical point of view. For example, when one wants to connect the set of admissible domains with vector fields, one has to suppose high smoothness conditions on the initial data in order to differentiate functions depending on the domain. Note also that to solve a conditional shape optimization problem by this method is yet more complicated and usually requires to reduce it to a non conditional problem (for example, by Lagrange's multipliers method). Moreover, the numerical implementation of the shape optimization problem requires to extend the vector field (obtained only on the boundary) to all the domain or to re-mesh at each iteration of the process, and both approaches are expensive (see for example Allaire [2]). In this paper, in order to avoid part of the above issues, we define and use another way of variation of domains, a way that is linked to the convexity context and based on the Minkowski sum. Recall that for any convex bounded domain the support function of this domain is a continuous convex and positive homogeneous function. Conversely, it is known that each continuous convex and positive homogeneous function is the support function of a convex bounded set (sub-differential of this function at the origin). Using this, the variation of the domain is clearly characterized by the variation of the corresponding support function. Moreover, according to the works $[6-8,24]$, one can express the shape derivative of volume cost functionals by means of support functions, and when solving problems numerically one gets a support function at each step of the implementation, the domain being recovered as the subdifferential of the support function. This is why we think that, in the context of convexity and numerical approximation, the use of support functions is more advantageous than that of vectors fields.

More precisely, we are interested in this paper in a shape optimization problem for solving numerically the so-called Bernoulli's free boundary problem or Bernoulli's problem. Let us note that many applications to fluid dynamic and industrial application lead to such a free boundary problem. As an example, let us quote the problem where the design of an annular capacitor is required in which one of the plates is prescribed while the other must be determined, so that the intensity of the electrostatic field remains constant thereon. Depending on whether we describe the internal or external plate, we have an exterior or an interior Bernoulli problem. This class of problems has been extensively studied theoretically by many authors, see for example $[1,11,16,22]$ and references therein. A practical way to study this type of problem is to transform it into a shape optimization problem where one of the Dirichlet or Neumann boundary condition on the free boundary is included into a cost functional while the other boundary condition is considered as part of an appropriate state problem. The question of existence for such shape optimization formulations is studied for example in [17] or [9], using the $C^{2}$ or $C^{1}$ regularity of the free boundary. On the numerical level, approximations of such shape optimization formulations of this type of free boundary problem can be found in the literature, using techniques that are different from ours. For example, a variational approach to shape derivatives is introduced in [21], a fictitious domain approach for solving this free boundary problem is proposed in [18], a pseudo-solid approach for solving its discrete problem is introduced in [28], a shape optimization approach by tracking the Neumann data and using the lagrangian formulation is developed 
in [25] and an iterative method based on a level-set formulation and boundary element method is proposed in [5,23]. We also quote the recent work [4] where the authors consider shape optimization problems in convex geometry and use support functions to study them via spectral expansions. In this work, we propose a shape optimization formulation of this problem using a volume cost functional. We develop a method for the shape differentiability of this cost functional, and establish the expression for its shape derivative via support functions, using the formula of shape derivative with respect to convex domains proposed in $[6,7]$. Then, we propose a gradient method for solving the shape optimization problem. Thereby, during the numerical solving of such problem, we get at each step support functions instead of domains. In this process, the discretization of the state problem is done using the boundary element method [10], while in most of shape optimization approaches, finite element method has been employed for sensitivity computation. However, the approaches generally suffer certain drawbacks; indeed, in shape optimization, cumbersome parameterization of design domain is required and time consuming re-meshing task is also necessary. Finally, we give some numerical results showing the effectiveness of the proposed approach.

The outline of the paper is as follows. In the second section, we present the shape optimization formulation for the approximation of Bernoulli's problem. In the third section, we give some preliminary results on the existence of the shape derivative of the cost functional on the considered family of admissible domains and establish the expression for its shape derivative by means of support functions. In the fourth section, we propose an algorithm for the approximation of the problem, based on a gradient method combined with the boundary element discretization. Finally, in order to illustrate the main result of this paper, the last section is focused on some numerical simulation applied to practical situations.

\section{Shape optimization formulation of Bernoulli's problem}

Let $D$ be a fixed smooth convex bounded and open subset of $\mathbb{R}^{n}(n \geq 2)$. Let $\Omega$ be a doubly connected open subset of $D$ of class $C^{2}$, that is, $\Omega$ is of the form $\Omega=\Omega_{2} \backslash \Omega_{1}$, where $\Omega_{2}$ is a convex bounded and open subset of $D$ of class $C^{2}$ and $\Omega_{1}$ is a fixed, compact and connected subset of $\Omega_{2}$ of class $C^{2}$. We denote by $\Gamma_{1}$ the boundary of $\Omega_{1}$ which is also the fixed inside part of the boundary of $\Omega$ and by $\Gamma_{2}$ the boundary of $\Omega_{2}$ which is at the same time the outside free boundary of $\Omega$ (see Figure 2).

Let us consider the external free boundary Bernoulli problem which consists in finding the free boundary $\Gamma_{2}$ and the solution $u=u(x)$ of the problem

$$
(P)\left\{\begin{array}{lll}
-\Delta u=f & \text { in } & \Omega=\Omega_{2} \backslash \Omega_{1}, \\
u=\varphi_{1} & \text { on } & \Gamma_{1}=\partial \Omega_{1}, \\
u=\varphi_{2} & \text { on } & \Gamma_{2}=\partial \Omega_{2}, \\
\partial_{\nu_{2}} u=\partial_{\nu_{2}} G & \text { on } & \Gamma_{2},
\end{array}\right.
$$

where $f, \varphi_{1}, \varphi_{2}$ and $G$ are given functions, $\nu_{2}$ is the outward unit normal vector to $\Gamma_{2}$ and $\partial_{\nu_{2}} u=\left\langle\left.\nabla u\right|_{\Gamma_{2}}, \nu_{2}\right\rangle$, where $\langle\cdot, \cdot\rangle$ denotes the scalar product in $\mathbb{R}^{n}$.

Let us first remark that in the free boundary problem (1) two boundary conditions must be satisfied on $\Gamma_{2}$. This suggests to reformulate such a problem as a shape optimization one to be able to solve it numerically. This is not a new idea and we refer for example to $[9,17,21]$ where such a method is used. Here, it consists in finding $\Gamma_{2}$ and $u$ solution of the shape optimization problem 


$$
\left\{\begin{array}{l}
\text { Minimize } \mathcal{J}\left(\Omega, u_{\Omega}\right) \text { for all } \Omega \in \mathcal{O} \\
\text { where } \mathcal{J}\left(\Omega, u_{\Omega}\right)=\int_{\Gamma_{2}}\left(u_{\Omega}(x)-\varphi_{2}(x)\right)^{2} d \sigma \\
\text { and } u_{\Omega} \text { is solution of } \\
(P E)\left\{\begin{array}{lll}
-\Delta v=f & \text { in } & \Omega=\Omega_{2} \backslash \Omega_{1}, \\
v=\varphi_{1} & \text { on } & \Gamma_{1}=\partial \Omega_{1}, \\
\partial_{\nu_{2}} v=\partial_{\nu_{2}} G & \text { on } & \Gamma_{2}=\partial \Omega_{2},
\end{array}\right.
\end{array}\right.
$$

where the set of admissible domains $\mathcal{O}$ is defined by

$$
\begin{gathered}
\mathcal{O}=\left\{\Omega \subset \mathbb{R}^{n} ; \Omega=\Omega_{2} \backslash \Omega_{1}, \quad \Omega_{2} \in \mathcal{O}\left(\Omega_{1}, D\right)\right\}, \\
\mathcal{O}\left(\Omega_{1}, D\right)=\left\{\Omega \subset \mathbb{R}^{n} ; \Omega \in \mathcal{U}(D), \quad \Omega_{1} \subset \Omega, \text { and } \bar{\Omega} \subset D\right\},
\end{gathered}
$$

and $\mathcal{U}(D)$ is the set of convex subdomains of $D$ which are of class $C^{2}$.

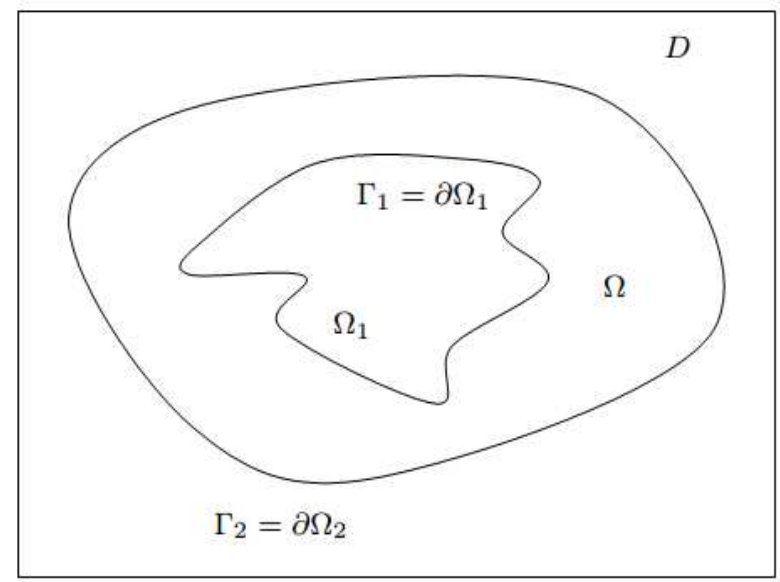

Figure 1: The considered domain $\Omega$.

However, tracking the Dirichlet boundary data at the free boundary $\Gamma_{2}$ of the state function $u$ in a boundary $L^{2}$-cost functional led us to some analytical issues that we have not been able to solve. Moreover, it was noticed by some authors that such an optimization formulation could be not well posed in some sense, see [15]. On the other hand, one can make the choice of tracking the Neumann data at the free boundary in an $L^{2}$-cost boundary functional while assuming that the state function $u$ satisfies a Dirichlet boundary value problem. The disadvantage in this case is that it requires high smoothness assumptions on the domains and on the state solution, even if such a formulation is rather well posed in the sense of [14]. In order to avoid the disadvantages related to the boundary integral cost functional, we propose here a new formulation using a domain integral cost functional. This also aims to use the shape derivative formulas developed in $[6,7]$.

\subsection{New formulation of the problem}

In order to propose another shape optimization formulation of (1), let us first give a preliminary result on convex domains.

Lemma 1. Let $\Omega$ be an open bounded convex subset of $\mathbb{R}^{n}$ of class $C^{k},(k \geq 1)$. Then, for all fixed $y \in \Omega$, we have

$$
\langle\nu(x), x-y\rangle>0, \quad \text { for all } \quad x \in \partial \Omega,
$$


where $\nu$ is the exterior unit normal vector to $\partial \Omega$. In particular, if $0 \in \Omega$, we get

$$
\langle\nu(x), x\rangle>0, \text { for all } x \in \partial \Omega \text {. }
$$

Proof. Let $\Omega$ be an open convex domain. Assume first that $0 \in \Omega$. According to [13], there exists a convex function $\varphi: \mathbb{R}^{n} \rightarrow \mathbb{R}$ such that

$$
\Omega=\left\{x \in \mathbb{R}^{n} / \varphi(x)<0\right\} \text { et } \partial \Omega=\left\{x \in \mathbb{R}^{n} / \varphi(x)=0\right\} .
$$

There exists also a neighborhood $W$ of $\Gamma$ such that $\varphi \in C^{k}(W), \quad \nabla \varphi \neq 0$ on $W$ and $\quad \nu=\nabla \varphi /\|\nabla \varphi\|$. If $x \in \partial \Omega$, by the characterization of the convexity at the points 0 and $x$ we have $\langle\nabla \varphi(x), 0-x\rangle \leq \varphi(0)-\varphi(x)$. Since $\varphi(0)<0$ and $\varphi(x)=0$, then, $\langle\nabla \varphi(x), x\rangle \geq-\varphi(0)>0$. Hence, multiplying by $\frac{1}{\| \nabla \varphi(x)}$ gives $\langle\nu(x), x\rangle>0$. Now, if $0 \notin \Omega$, we consider the translated $\Omega-y$ of $\Omega$ by means of a fixed $y \in \Omega$. Clearly, this is an open bounded convex subset of $\mathbb{R}^{n}$ of class $C^{k}$ containing 0 . Hence, by application of the above result, we obtain

$$
\left\langle\nu_{\Omega-y}(x), x\right\rangle>0, \quad \text { for all } \quad x \in \partial \Omega-y .
$$

Since $\nu_{\Omega-y}(x-y)=\nu(x)$ for all $x \in \partial \Omega$, we get $\quad\langle\nu(x), x-y\rangle>0, \quad$ for all $\quad x \in \partial \Omega$.

For simplicity, we shall assume in the rest of the paper that $0 \in \Omega_{1}$. If $\Omega=\Omega_{2} \backslash \Omega_{1}$ is as before, then, according to Lemma 1, we have

$$
\left(\nu_{2}(x), x\right)>0, \quad \text { for all } \quad x \in \Gamma_{2},
$$

and this suggests to consider the integral

$$
\int_{\Gamma_{2}}\left(u_{\Omega}(x)-\varphi_{2}(x)\right)^{2}\left\langle\nu_{2}(x), x\right\rangle d \sigma
$$

instead of that in (2). In what follows, we are going to transform this boundary integral into a domain one.

To do so, let us first recall that, since the domain $\Omega$ is doubly connected, the exterior unit normal vector to $\Omega$ is given by

$$
\begin{aligned}
\nu: \partial \Omega & \longrightarrow S^{n-1} \\
x & \longmapsto \nu(x)= \begin{cases}\nu_{2}(x) & \text { si } x \in \Gamma_{2} \\
-\nu_{1}(x) & \text { si } x \in \Gamma_{1} .\end{cases}
\end{aligned}
$$

where $\nu_{k}$, for $k=1,2$ is the exterior unit normal vector to $\Omega_{k}$ and $S^{n-1}$ is the unit sphere in $\mathbb{R}^{n}$. If $u_{\Omega}$ the solution of $(P E)$ associated to $\Omega=\Omega_{2} \backslash \Omega_{1}$, we can write

$$
\begin{aligned}
& \int_{\Gamma_{2}}\left(u_{\Omega}(x)-\varphi_{2}(x)\right)^{2}\left\langle\nu_{2}(x), x\right\rangle d \sigma=\int_{\Gamma_{2}}\left(u_{\Omega}(x)-\varphi_{2}(x)\right)^{2}\langle\nu(x), x\rangle d \sigma \\
& +\int_{\Gamma_{1}}\left(u_{\Omega}(x)-\varphi_{2}(x)\right)^{2}\langle\nu(x), x\rangle d \sigma-\int_{\Gamma_{1}}\left(u_{\Omega}(x)-\varphi_{2}(x)\right)^{2}\langle\nu(x), x\rangle d \sigma \\
& =\int_{\Gamma}\left(u_{\Omega}(x)-\varphi_{2}(x)\right)^{2}\langle\nu(x), x\rangle d \sigma+\int_{\Gamma_{1}}\left(u_{\Omega}(x)-\varphi_{2}(x)\right)^{2}\left\langle\nu_{1}(x), x\right\rangle d \sigma \\
& =\int_{\Omega} \operatorname{div}\left(\left(u_{\Omega}(x)-\varphi_{2}(x)\right)^{2} x\right) d x+\int_{\Gamma_{1}}\left(\varphi_{1}(x)-\varphi_{2}(x)\right)^{2}\left\langle\nu_{1}(x), x\right\rangle d \sigma,
\end{aligned}
$$

where $\operatorname{div}(v)$ denotes the divergence of the vector $v$ and we have applied of course the divergence formula and the fact that $u_{\Omega}=\varphi_{1}$ on $\Gamma_{1}$. Thus, we have

$$
\int_{\Omega} \operatorname{div}\left(\left(u_{\Omega}(x)-\varphi_{2}(x)\right)^{2} x\right) d x=\int_{\Gamma_{2}}\left(u_{\Omega}(x)-\varphi_{2}(x)\right)^{2}\left\langle\nu_{2}(x), x\right\rangle d \sigma-\int_{\Gamma_{1}}\left(\varphi_{1}(x)-\varphi_{2}(x)\right)^{2}\left\langle\nu_{1}(x), x\right\rangle d \sigma .
$$


Since the term $\int_{\Gamma_{1}}\left(\varphi_{1}(x)-\varphi_{2}(x)\right)^{2}\left\langle\nu_{1}(x),(x)\right\rangle d \sigma$ do not depend on the variable domain $\Omega_{2}$, minimizing (3) is equivalent to minimizing the domain integral (4). Therefore, we can propose as a shape optimization formulation for the approximation of the free boundary problem (1), the following one

$$
(P O)\left\{\begin{array}{l}
\text { Minimize } \mathcal{J}\left(\Omega, u_{\Omega}\right) \text { for all } \Omega \in \mathcal{O} \\
\text { where } \mathcal{J}\left(\Omega, u_{\Omega}\right)=\int_{\Omega} \operatorname{div}\left(\left(u_{\Omega}(x)-\varphi_{2}(x)\right)^{2} x\right) d x \\
\text { and } u_{\Omega} \text { is solution of } \\
(P E)\left\{\begin{array}{lll}
-\Delta v=f & \text { in } & \Omega=\Omega_{2} \backslash \Omega_{1}, \\
v=\varphi_{1} & \text { on } & \Gamma_{1}, \\
\partial_{\nu_{2}} v=\partial_{\nu_{2}} G & \text { on } & \Gamma_{2} .
\end{array}\right.
\end{array}\right.
$$

In what follows, we propose a numerical method of the approximation of this problem based on a gradient method. This requires to study the first variation or differentiability of the cost functional with respect to the family of domains $\mathcal{O}$. For this, we shall adapt the formulas proposed in $[6,7]$ to be able to express the shape derivative of the cost functional by means of support functions.

\section{Shape derivative of the cost functional}

In order to obtain an expression of the shape derivative of the cost functional for the shape optimization problem (5), we first recall the shape derivative formulas established in $[6,7]$ in the case of convex domains and then adapt them to our class $\mathcal{O}$ of doubly connected domains.

Let us start by recalling the notion of support function of a convex bounded domain. See [27] for more details. To any bounded convex domain $\Omega$, one can associate its support function defined by

$$
P_{\Omega}: x \in \mathbb{R}^{n} \mapsto P_{\Omega}(x)=\sup _{y \in \Omega}\langle x, y\rangle \in \mathbb{R},
$$

which is a continuous, convex and positively homogeneous function. The converse is also true: for any continuous, convex, positively homogeneous function $P$ there exists a unique convex bounded open set $\Omega$, such that $P$ is its support function, that is, $P=P_{\Omega}$. Recall also that $\Omega$ is then obtained via the sub-differential of $P$ at the origin:

$$
\bar{\Omega}=\partial P(0):=\left\{\xi \in \mathbb{R}^{n} /\langle\xi, y\rangle \leq P(y), \forall y \in \mathbb{R}^{n}\right\} .
$$

In $[6,7]$, the authors used the deformations $\Omega_{\varepsilon}=\Omega_{0}+\varepsilon \Omega$ (Minkowski deformation) and $\Omega_{\varepsilon}^{\prime}=(1-\varepsilon) \Omega_{0}+$ $\varepsilon \Omega$ (Niftiyev-Gasimov deformation), $\varepsilon \in[0,1]$, and compute the shape derivatives of the volume integral shape functional

$$
\Omega \mapsto J(\Omega)=\int_{\Omega} f(x) d x
$$

where $f \in W_{l o c}^{1,1}\left(\mathbb{R}^{n}\right)$. They obtained the following formulas:

Theorem 1. Let $\Omega_{0}$ and $\Omega$ be convex and bounded domains in $\mathbb{R}^{n}$. Then, we have

$$
\lim _{\varepsilon \rightarrow 0^{+}} \frac{J\left(\Omega_{\varepsilon}\right)-J\left(\Omega_{0}\right)}{\varepsilon}=\int_{\partial \Omega_{0}} f(x) P_{\Omega}\left(\nu_{0}(x)\right) d \sigma(x),
$$

and

$$
\lim _{\varepsilon \rightarrow 0^{+}} \frac{J\left(\Omega_{\varepsilon}^{\prime}\right)-J\left(\Omega_{0}\right)}{\varepsilon}=\int_{\partial \Omega_{0}} f(x)\left(P_{\Omega}\left(\nu_{0}(x)\right)-P_{\Omega_{0}}\left(\nu_{0}(x)\right)\right) d \sigma(x),
$$

where $\nu_{0}$ denotes the exterior unit normal vector to $\Omega_{0}$. 
The compact and connected set $\Omega_{1}$ being fixed in $\mathbb{R}^{n}$ and of class $C^{2}$, recall that $\mathcal{O}\left(\Omega_{1}, D\right)$ denotes the set of all $C^{2}$ open convex and bounded subsets $\Omega_{2}$ of $\mathbb{R}^{n}$ such that $\Omega_{1} \subset \Omega_{2}$ and $\bar{\Omega}_{2} \subset D, D$ being also fixed and is in fact a large smooth convex and bounded domain. We shall also denote by $\mathcal{U}(D)$ the set of convex subdomains of $D$ which are of class $C^{2}$.

Now, if $\varepsilon \in[0,1], \Omega_{2} \in \mathcal{O}\left(\Omega_{1}, D\right), \Omega_{2}^{\prime} \in \mathcal{U}(D)$ and if $\varepsilon$ is small enough, we have $\Omega_{2}+\varepsilon \Omega_{2}^{\prime} \in \mathcal{O}\left(\Omega_{1}, D\right)$ and we can write obviously

$$
J\left(\left(\Omega_{2}+\varepsilon \Omega_{2}^{\prime}\right) \backslash \Omega_{1}\right)=\int_{D} \chi\left(\Omega_{2}+\varepsilon \Omega_{2}^{\prime}\right) \backslash \Omega_{1} f(x) d x=J\left(\Omega_{2}+\varepsilon \Omega_{2}^{\prime}\right)-J\left(\Omega_{1}\right) .
$$

Hence, applying Theorem 1 yields

$$
\left.\frac{d}{d \varepsilon}\left(J\left(\Omega_{2}+\varepsilon \Omega_{2}^{\prime} \backslash \Omega_{1}\right)\right)\right|_{\varepsilon=0^{+}}=\left.\frac{d}{d \varepsilon}\left(J\left(\Omega_{2}+\varepsilon \Omega_{2}^{\prime}\right)\right)\right|_{\varepsilon=0^{+}}=\int_{\Gamma_{2}} f(x) P_{\Omega_{2}^{\prime}}\left(\nu_{2}(x)\right) d \sigma(x) .
$$

A straightforward consequence of Theorem 1 is then

Corollary 1. Let $\Omega_{2} \in \mathcal{O}\left(\Omega_{1}, D\right), \Omega_{2}^{\prime} \in \mathcal{U}(D)$. Then, we have

$$
\lim _{\varepsilon \rightarrow 0^{+}} \frac{J\left(\left(\Omega_{2}+\varepsilon \Omega_{2}^{\prime}\right) \backslash \Omega_{1}\right)-J\left(\Omega_{2} \backslash \Omega_{1}\right)}{\varepsilon}=\int_{\Gamma_{2}} f(x) P_{\Omega_{2}^{\prime}}\left(\nu_{2}(x)\right) d \sigma(x),
$$

where $\nu_{2}$ denotes the exterior unit normal vector to $\Omega_{2}$.

This is why we shall use $\left(\Omega_{2}+\varepsilon \Omega_{2}^{\prime}\right) \backslash \Omega_{1}$ as the deformation of the admissible set $\Omega_{2} \backslash \Omega_{1}$. Note that this is equivalent to the deformation of the exterior boundary of $\Omega_{2} \backslash \Omega_{1}$. Of course, the above limit is nothing but the shape derivative of $J$ at $\Omega_{2} \backslash \Omega_{1} \in \mathcal{O}$ in the direction $\Omega_{2}^{\prime}$.

In the situation where the function $f$ depends on domains, we have the following formula:

Proposition 1. Let $f$ and $f_{\varepsilon}, \varepsilon \in[0,1]$, be functions in $L^{1}(D)$ such that $f_{0} \in W^{1,1}(D)$ and $f=\lim _{\varepsilon \rightarrow 0^{+}} \frac{1}{\varepsilon}\left(f_{\varepsilon}-f_{0}\right)$ in $L^{1}(D)$. If $\Omega_{2} \in \mathcal{O}\left(\Omega_{1}, D\right)$ and $\Omega_{2}^{\prime} \in \mathcal{U}(D)$, define $I(\varepsilon), \varepsilon \in[0,1]$, by

$$
I(\varepsilon)=\int_{\left(\Omega_{2}+\varepsilon \Omega_{2}^{\prime}\right) \backslash \Omega_{1}} f_{\varepsilon}(x) d x .
$$

Then, we have

$$
\lim _{\varepsilon \rightarrow 0^{+}} \frac{I(\varepsilon)-I(0)}{\varepsilon}=\int_{\Omega_{2} \backslash \Omega_{1}} f(x) d x+\int_{\Gamma_{2}} f_{0}(x) P_{\Omega_{2}^{\prime}}\left(\nu_{2}(x)\right) d \sigma(x) .
$$

where $\nu_{2}$ denotes the exterior unit normal vector to $\Omega_{2}$.

Proof. First, we can write $I(\varepsilon)=\int_{\Omega_{2}+\varepsilon \Omega_{2}^{\prime}} f_{\varepsilon}(x) d x-\int_{\Omega_{1}} f_{\varepsilon}(x) d x$ and, clearly, we have

$$
\left|\int_{\Omega_{1}}\left(\frac{1}{\varepsilon}\left(f_{\varepsilon}-f_{0}\right)-f\right) d x\right| \leq \int_{D}\left|\frac{1}{\varepsilon}\left(f_{\varepsilon}-f_{0}\right)-f\right| d x \rightarrow 0
$$

as $\varepsilon \rightarrow 0^{+}$, which reduces the study to the term $\int_{\Omega_{2}+\varepsilon \Omega_{2}^{\prime}} f_{\varepsilon}(x) d x$ to which we can apply Corollary 1 of [6] to obtain $\lim _{\varepsilon \rightarrow 0^{+}} \frac{I(\varepsilon)-I(0)}{\varepsilon}=\int_{\Omega_{2}} f(x) d x+\int_{\Gamma_{2}} f_{0}(x) P_{\Omega_{2}^{\prime}}\left(\nu_{2}(x)\right) d \sigma(x)-\int_{\Omega_{1}} f(x) d x=\int_{\Omega_{2} \backslash \Omega_{1}} f(x) d x+\int_{\Gamma_{2}} f_{0}(x) P_{\Omega_{2}^{\prime}}\left(\nu_{2}(x)\right) d \sigma(x)$

which ends the proof.

In the case where $f$ is the product of two functions depending on the domains, one can state the following: 
Corollary 2. Let $\left(f_{\varepsilon}\right)_{\varepsilon \in] 0,1[}$ and $\left(g_{\varepsilon}\right)_{\varepsilon \in] 0,1[}$ be two families of functions in $L^{2}(D)$ and let $f_{0} \in H^{1}(D), g_{0} \in H^{1}(D)$ and $f, g$ be functions such that $f=\lim _{\varepsilon \rightarrow 0^{+}} \frac{1}{\varepsilon}\left(f_{\varepsilon}-f_{0}\right)$ in $L^{2}(D)$ and $g=\lim _{\varepsilon \rightarrow 0^{+}} \frac{1}{\varepsilon}\left(g_{\varepsilon}-g_{0}\right)$ in $L^{2}(D)$. Consider the function

$$
\varepsilon \in] 0,1\left[\mapsto F(\varepsilon)=\int_{\left(\Omega_{2}+\varepsilon \Omega_{2}^{\prime}\right) \backslash \Omega_{1}} f_{\varepsilon} g_{\varepsilon}(x) d x \in \mathbb{R} .\right.
$$

Then, we have

$$
\lim _{\varepsilon \rightarrow 0^{+}} \frac{F(\varepsilon)-F(0)}{\varepsilon}=\int_{\Omega_{2} \backslash \Omega_{1}}\left(g f_{0}+f g_{0}\right)(x) d x+\int_{\Gamma_{2}} f_{0} g_{0}(x) P_{\Omega_{2}^{\prime}}\left(\nu_{2}(x)\right) d \sigma(x),
$$

where $\nu_{2}$ denotes the exterior unit normal vector to $\Omega_{2}$.

Proof. This is an easy consequence of Proposition 1 since $f_{0} g_{0} \in W^{1,1}(D)$ and

$$
\frac{1}{\varepsilon}\left(f_{\varepsilon} g_{\varepsilon}-f_{0} g_{0}\right)-f_{0} g-f g_{0}=\frac{1}{\varepsilon}\left(f_{\varepsilon}-f_{0}\right)\left(g_{\varepsilon}-g_{0}\right)+g_{0}\left(\frac{1}{\varepsilon}\left(f_{\varepsilon}-f_{0}\right)-f\right)+f_{0}\left(\frac{1}{\varepsilon}\left(g_{\varepsilon}-g_{0}\right)-g\right) \underset{\varepsilon \rightarrow 0^{+}}{\longrightarrow} 0
$$

in $L^{1}(D)$.

\section{Shape derivative of the shape optimization problem}

Recall that $\mathcal{U}(D)$ is the set of convex subdomains of $D$ which are of class $C^{2}$,

$$
\begin{aligned}
\mathcal{O}\left(\Omega_{1}, D\right)= & \left\{\Omega \subset \mathbb{R}^{n} ; \Omega_{1} \subset \Omega, \bar{\Omega} \subset D, \Omega \text { is open, convex and of class } C^{2}\right\}, \\
& \text { and } \mathcal{O}=\left\{\Omega \subset \mathbb{R}^{n} ; \Omega=\Omega_{2} \backslash \Omega_{1}, \quad \Omega_{2} \in \mathcal{O}\left(\Omega_{1}, D\right)\right\}
\end{aligned}
$$

where $\Omega_{1}$ is a fixed compact and connected subset of $\mathbb{R}^{n}$ of class $C^{2}$ and $D$ is a fixed large smooth convex and bounded domain in $\mathbb{R}^{n}$.

In this section, we state and prove the main theoretical result of this paper which concerns the shape derivative of the functional

$$
\mathcal{J}\left(\Omega, u_{\Omega}\right)=\int_{\Omega} \operatorname{div}\left(\left(u_{\Omega}(x)-\varphi_{2}(x)\right)^{2} x\right) d x
$$

where $\Omega \in \mathcal{O}$ and $u_{\Omega}$ is the solution of the state problem on $\Omega$, that is,

$$
\left\{\begin{array}{lll}
-\Delta u_{\Omega}=f & \text { in } & \Omega=\Omega_{2} \backslash \Omega_{1} \\
u_{\Omega}=\varphi_{1} & \text { on } & \Gamma_{1}=\partial \Omega_{1} \\
\partial_{\nu_{2}} u_{\Omega}=\partial_{\nu_{2}} G & \text { on } & \Gamma_{2}=\partial \Omega_{2} .
\end{array}\right.
$$

The following remark will be useful in the proof of the theorem below.

Remark 1. When one assumes that $\Omega$ is strongly convex, $\Omega^{\varepsilon}$ can be considered as a deformation of the domain $\Omega$ by a vector field $V(x)$ satisfying $\langle V(x), \nu(x)\rangle=P_{\Omega^{\prime}}(\nu(x))$ (see $\left.[6,7]\right)$. Therefore, when $f \in H^{1}(D), \varphi_{1} \in H^{\frac{1}{2}}\left(\Gamma_{1}\right)$ and $G \in H^{3}(D)$, we can write

$$
\widetilde{u}_{\varepsilon}=\widetilde{u}+\varepsilon u^{\prime}+\varepsilon v_{\varepsilon} \text { in } H^{1}(D)
$$

where $\widetilde{u}$ and $\widetilde{u}_{\varepsilon}$ are extensions to $D$ of $u_{\Omega}$ and $u_{\Omega_{\varepsilon}}$ respectively, and $u^{\prime}$ is the shape derivative of $\widetilde{u}$ with respect to the vector field $V$ (see for example, $[13,26]$ ). It follows from that result that there exists a function denoted $F_{0}^{\prime}$ in $L^{1}(D)$, such that

$$
\frac{1}{\varepsilon}\left[F\left(., \widetilde{u}_{\varepsilon}, \nabla \widetilde{u}_{\varepsilon}\right)-F(., \widetilde{u}, \nabla \widetilde{u})\right]-F_{0}^{\prime} \longrightarrow 0 \quad \text { in } \quad L^{1}(D), \varepsilon \rightarrow 0,
$$

which allows to apply the Proposition 1 in order to compute the shape derivative of $\mathcal{J}$. 
We can state now the main theoretical result of this paper.

Theorem 2. In the formulation (5), assume that $f \in H^{1}(D), \varphi_{2} \in H^{2}(D), \varphi_{1} \in H^{\frac{1}{2}}\left(\Gamma_{1}\right)$ and $G \in H^{3}(D)$. Let $\Omega_{2} \in \mathcal{O}\left(\Omega_{1}, D\right), \Omega_{2}^{\prime} \in \mathcal{U}(D)$ and $\Omega^{\varepsilon}=\left(\Omega_{2}+\varepsilon \Omega_{2}^{\prime}\right) \backslash \Omega_{1}$. Assume further that $0 \in \Omega_{2}^{\prime}$ and that $\Omega_{2}^{\prime}$ is strongly convex. Then, the shape derivative of $\mathcal{J}\left(\Omega, u_{\Omega}\right)$ at $\Omega=\Omega_{2} \backslash \Omega_{1}$ in the direction $\Omega_{2}^{\prime}$ is given by

$$
\lim _{\varepsilon \rightarrow 0^{+}} \frac{\mathcal{J}\left(\Omega^{\varepsilon}, u_{\Omega^{\varepsilon}}\right)-\mathcal{J}\left(\Omega, u_{\Omega}\right)}{\varepsilon}=\int_{\Gamma_{2}} \mathcal{B}(x) P_{\Omega_{2}^{\prime}}\left(\nu_{2}(x)\right) d \sigma(x),
$$

where

$$
\mathcal{B}=\operatorname{div}\left(\left(u_{\Omega}-\varphi_{2}\right)^{2} I d\right)+\left\langle\nabla\left(u_{\Omega}-G\right), \nabla \psi\right\rangle-(f+\Delta G) \psi
$$

$\psi$ is the solution of the adjoint state problem

$$
\begin{cases}-\Delta \psi=0 & \text { in } \quad \Omega, \\ \psi=0 & \text { on } \quad \Gamma_{1}=\partial \Omega_{1}, \\ \partial_{\nu_{2}} \psi=-2\left(u_{\Omega}-\varphi_{2}\right)\left\langle\nu_{2}, I d\right\rangle & \text { on } \quad \Gamma_{2}=\partial \Omega_{2},\end{cases}
$$

and $\nu_{2}$ denotes the exterior unit normal vector to $\Gamma_{2}$.

Proof. The fact that $0 \in \Omega_{2}^{\prime}$ implies that $\Omega_{2} \subset \Omega_{2}+\varepsilon \Omega_{2}^{\prime}$ and hence that $\Omega=\Omega_{2} \backslash \Omega_{1} \subset \Omega^{\varepsilon}=\left(\Omega_{2}+\varepsilon \Omega_{2}^{\prime}\right) \backslash \Omega_{1}$. Consequently, we obtain that $u_{\Omega}$ and $u_{\Omega^{\varepsilon}}$ are respectively solutions of

$$
\left\{\begin{array} { l l } 
{ - \Delta u _ { \Omega } = f } & { \text { in } \quad \Omega , } \\
{ u _ { \Omega } = \varphi _ { 1 } } & { \text { on } \quad \Gamma _ { 1 } , }
\end{array} \quad \left\{\begin{array}{ll}
-\Delta u_{\Omega^{\varepsilon}}=f & \text { in } \quad \Omega, \\
u_{\Omega^{\varepsilon}}=\varphi_{1} & \text { on } \Gamma_{1} .
\end{array}\right.\right.
$$

Therefore, if we denote by $\delta u=u_{\Omega^{\varepsilon}}-u_{\Omega}$, we obtain that $\delta u$ satisfies

$$
\begin{cases}-\Delta(\delta u)=0 & \text { in } \quad \Omega, \\ \delta u=0 & \text { on } \quad \Gamma_{1} \subseteq \Gamma .\end{cases}
$$

Now, since $\operatorname{div}\left(\left(u_{\Omega}-\varphi_{2}\right)^{2} x\right)=2\left(u_{\Omega}-\varphi_{2}\right)\left\langle\nabla\left(u_{\Omega}-\varphi_{2}\right), x\right\rangle+n\left(u_{\Omega}-\varphi_{2}\right)^{2}$, let us introduce the function $F$ defined on $D \times \mathbb{R} \times \mathbb{R}^{n}$ by $\quad F(x, y, z)=2\left(y-\varphi_{2}(x)\right)\left\langle z-\nabla \varphi_{2}(x), x\right\rangle+n\left(y-\varphi_{2}(x)\right)^{2}, \quad$ so that we can write

$$
\mathcal{J}\left(\Omega, u_{\Omega}\right)=\int_{\Omega} F\left(x, u_{\Omega}(x), \nabla u_{\Omega}(x)\right) d x .
$$

Next, consider the expression $\triangle \mathcal{J}=\mathcal{J}\left(\Omega^{\varepsilon}, u_{\Omega^{\varepsilon}}\right)-\mathcal{J}\left(\Omega, u_{\Omega}\right)$. We can write

$$
\triangle \mathcal{J}=\int_{\Omega}\left(F\left(x, u_{\Omega^{\varepsilon}}(x), \nabla u_{\Omega^{\varepsilon}}(x)\right)-F\left(x, u_{\Omega}(x), \nabla u_{\Omega}(x)\right)\right) d x+J_{1}(\varepsilon) .
$$

where

$$
J_{1}(\varepsilon)=\int_{\Omega^{\varepsilon}} F\left(x, u_{\Omega^{\varepsilon}}(x), \nabla u_{\Omega^{\varepsilon}}(x)\right) d x-\int_{\Omega} F\left(x, u_{\Omega^{\varepsilon}}(x), \nabla u_{\Omega^{\varepsilon}}(x)\right) d x .
$$

Let us now rewrite the expression $\delta F=F\left(x, u_{\Omega_{\varepsilon}}, \nabla u_{\Omega_{\varepsilon}}\right)-F\left(x, u_{\Omega}, \nabla u_{\Omega}\right)$ as follows:

$$
\begin{aligned}
\delta F & =\operatorname{div}\left(\left(u_{\Omega_{\varepsilon}}-\varphi_{2}\right)^{2} I_{d}\right)-\operatorname{div}\left(\left(u_{\Omega}-\varphi_{2}\right)^{2} I_{d}\right)=\operatorname{div}\left(\delta u\left(\delta u+2\left(u_{\Omega}-\varphi_{2}\right)\right) I_{d}\right) \\
& =\left\langle\nabla(\delta u), I_{d}\right\rangle\left(\delta u+2\left(u_{\Omega}-\varphi_{2}\right)\right)+\delta u\left\langle\nabla\left(\delta u+2\left(u_{\Omega}-\varphi_{2}\right)\right), I_{d}\right\rangle+n \delta u\left(\delta u+2\left(u_{\Omega}-\varphi_{2}\right)\right) \\
& =2 \delta u\left\langle\nabla(\delta u), I_{d}\right\rangle+2 \delta u\left\langle\nabla\left(u_{\Omega}-\varphi_{2}\right), I_{d}\right\rangle+2\left(u_{\Omega}-\varphi_{2}\right)\left\langle\nabla(\delta u), I_{d}\right\rangle+n(\delta u)^{2}+2 n \delta u\left(u_{\Omega}-\varphi_{2}\right) \\
& =\left\langle v_{\Omega}, \nabla(\delta u)\right\rangle+\operatorname{div}\left(v_{\Omega}\right) \delta u+n(\delta u)^{2}+2 \delta u(x)\langle\nabla(\delta u)(x), x\rangle \\
& =\operatorname{div}\left(v_{\Omega} \delta u\right)+n(\delta u)^{2}+2 \delta u(x)\langle\nabla(\delta u)(x), x\rangle .
\end{aligned}
$$


where $v_{\Omega}(x)=2\left(u_{\Omega}(x)-\varphi_{2}(x)\right) x$. Hence,

$$
\triangle \mathcal{J}=J_{1}(\varepsilon)+J_{2}(\varepsilon)+J_{3}(\varepsilon)
$$

where

$$
J_{2}(\varepsilon)=\int_{\Omega} \operatorname{div}\left(v_{\Omega} \delta u\right)(x) d x \quad \text { and } \quad J_{3}(\varepsilon)=n\|\delta u\|_{L^{2}(\Omega)}^{2}+2 \int_{\Omega} \delta u(x)\langle\nabla(\delta u)(x), x\rangle d x .
$$

It follows from the divergence formula that

$$
J_{2}(\varepsilon)=\int_{\partial \Omega}\left\langle v_{\Omega}, \nu\right\rangle(x) \delta u(x) d \sigma=2 \int_{\Gamma_{2}}\left(u_{\Omega}-\varphi_{2}\right)\left\langle\nu_{2}(x), x\right\rangle \delta u(x) d \sigma-2 \int_{\Gamma_{1}}\left(u_{\Omega}-\varphi_{2}\right)\left\langle\nu_{1}(x), x\right\rangle \delta u(x) d \sigma,
$$

and if $\psi$ is the unique solution of the boundary value problem

$$
\begin{cases}-\Delta \psi=0 & \text { in } \Omega, \\ \psi=0 & \text { on } \Gamma_{1}, \\ \partial_{\nu_{2}} \psi=-2\left(u_{\Omega}-\varphi_{2}\right)\left\langle\nu_{2}, I d\right\rangle & \text { on } \Gamma_{2},\end{cases}
$$

we can write

$$
J_{2}(\varepsilon)=-\int_{\Gamma_{2}} \partial_{\nu_{2}} \psi(x) \delta u(x) d \sigma-2 \int_{\Gamma_{1}}\left(u_{\Omega}-\varphi_{2}\right)\left\langle\nu_{1}(x), x\right\rangle \delta u(x) d \sigma=-\int_{\Gamma_{2}} \partial_{\nu_{2}} \psi(x) \delta u(x) d \sigma(x),
$$

since $\delta u=0$ on $\Gamma_{1}$. Using the fact that $\Delta(\delta u)=\Delta \psi=0$ on $\Omega$ and $\delta u=\psi=0$ on $\Gamma_{1}$, it follows from the Green's formula that

$$
J_{2}(\varepsilon)=-\int_{\Gamma} \partial_{\nu} \psi(x) \delta u(x) d \sigma=-\int_{\Gamma} \psi(x) \partial_{\nu} \delta u(x) d \sigma .
$$

On the other hand, $J_{1}$ can be written $\quad J_{1}(\varepsilon)=J_{1,1}(\varepsilon)-J_{1,2}(\varepsilon) \quad$ with

$$
J_{1,1}(\varepsilon)=\int_{\Omega_{\varepsilon}} F\left(x, u_{\Omega_{\varepsilon}}(x), \nabla u_{\Omega_{\varepsilon}}(x)\right) d x \quad \text { and } \quad J_{1,2}(\varepsilon)=\int_{\Omega} F\left(x, u_{\Omega_{\varepsilon}}(x), \nabla u_{\Omega_{\varepsilon}}(x)\right) d x .
$$

Now, it follows from Remark 1 that one can apply Proposition 1 to the function $F_{\varepsilon}=F\left(., u_{\Omega_{\varepsilon}}, \nabla u_{\Omega_{\varepsilon}}\right)$ to obtain

$$
\left.\frac{d}{d \varepsilon}\left(J_{1,1}(\varepsilon)\right)\right|_{\varepsilon=0^{+}}=\int_{\Omega_{2} \backslash \Omega_{1}} F_{0}^{\prime}(x) d x+\int_{\Gamma_{2}} F_{0}(x) P_{\Omega_{2}^{\prime}}\left(\nu_{2}(x)\right) d \sigma \quad \text { and }\left.\quad \frac{d}{d \varepsilon}\left(J_{1,2}(\varepsilon)\right)\right|_{\varepsilon=0^{+}}=\int_{\Omega_{2} \backslash \Omega_{1}} F_{0}^{\prime}(x) d x .
$$

Hence,

$$
\left.\frac{d}{d \varepsilon}\left(J_{1}(\varepsilon)\right)\right|_{\varepsilon=0^{+}}=\int_{\Gamma_{2}} F_{0}(x) P_{\Omega_{2}^{\prime}}\left(\nu_{2}(x)\right) d \sigma, \text { where } \quad F_{0}=F\left(., u_{\Omega}, \nabla u_{\Omega}\right) .
$$

Next, let's calculate the derivative of $J_{2}$. We can write

$$
J_{2}(\varepsilon)=-\int_{\Gamma} \partial_{\nu} \delta u(x) \psi(x) d \sigma=-\int_{\Gamma}\left(\partial_{\nu} u_{\Omega^{\varepsilon}}(x)-\partial_{\nu} G(x)\right) \psi(x) d \sigma+\int_{\Gamma}\left(\partial_{\nu} u_{\Omega}(x)-\partial_{\nu} G(x)\right) \psi(x) d \sigma
$$

First, since $\partial_{\nu_{2}} u_{\Omega}=\partial_{\nu_{2}} G$ on $\Gamma_{2}$ and $\psi=0$ on $\Gamma_{1}$, we have

$$
\int_{\Gamma}\left(\partial_{\nu} u_{\Omega}(x)-\partial_{\nu} G(x)\right) \psi(x) d \sigma=\int_{\Gamma_{1}}\left(\partial_{\nu_{1}} u_{\Omega}-\partial_{\nu_{1}} G\right)(x) \psi(x) d \sigma+\int_{\Gamma_{2}}\left(\partial_{\nu_{2}} u_{\Omega}-\partial_{\nu_{2}} G\right)(x) \psi(x) d \sigma=0 .
$$

Next, it follows from the fact that $\partial_{\nu_{2}^{\varepsilon}} u_{\Omega^{\varepsilon}}=\partial_{\nu_{2}^{\varepsilon}} G$ on $\partial\left(\Omega_{2}+\varepsilon \Omega_{2}^{\prime}\right)$ and $\psi=0$ on $\Gamma_{1}$, that $\left(\partial_{\nu^{\varepsilon}} u_{\Omega^{\varepsilon}}-\partial_{\nu^{\varepsilon}} G\right) \psi=0$ on $\Gamma^{\varepsilon}=\partial \Omega^{\varepsilon}$. Therefore, we can write

$$
J_{2}(\varepsilon)=-\int_{\Gamma}\left(\partial_{\nu} u_{\Omega^{\varepsilon}}-\partial_{\nu} G\right) \psi d \sigma+\int_{\Gamma^{\varepsilon}}\left(\partial_{\nu^{\varepsilon}} u_{\Omega^{\varepsilon}}-\partial_{\nu^{\varepsilon}} G\right) \psi d \sigma,
$$

and it follows from the divergence formula that

$$
J_{2}(\varepsilon)=-\int_{\Omega} \operatorname{div}\left(\nabla\left(u_{\Omega^{\varepsilon}}-G\right) \psi\right)(x) d x+\int_{\Omega^{\varepsilon}} \operatorname{div}\left(\nabla\left(u_{\Omega^{\varepsilon}}-G\right) \psi\right)(x) d x .
$$


Now, since $-\Delta u_{\Omega_{\varepsilon}}=f \quad$ on $\quad \Omega_{\varepsilon}$ and $\Omega \subset \Omega_{\varepsilon}$, we also have $-\Delta u_{\Omega_{\varepsilon}}=f$ on $\Omega$. Hence,

$$
\operatorname{div}\left(\nabla\left(u_{\Omega_{\varepsilon}}-G\right) \psi\right)=\left\langle\nabla\left(u_{\Omega_{\varepsilon}}-G\right), \nabla \psi\right\rangle+\psi \Delta\left(u_{\Omega_{\varepsilon}}-G\right)=\left\langle\nabla\left(u_{\Omega_{\varepsilon}}-G\right), \nabla \psi\right\rangle+\psi(-f-\Delta G) .
$$

Let us write $\quad J_{2}(\varepsilon)=J_{2,1}(\varepsilon)+J_{2,2}(\varepsilon)+J_{2,3}(\varepsilon)+J_{2,4}(\varepsilon)$, where

$$
\begin{aligned}
& J_{2,1}(\varepsilon)=-\int_{\Omega}\left\langle\nabla\left(u_{\Omega_{\varepsilon}}-G\right), \nabla \psi\right\rangle(x) d x, \quad J_{2,2}(\varepsilon)=-\int_{\Omega}(-f-\Delta G) \psi(x) d x, \\
& J_{2,3}(\varepsilon)=\int_{\Omega_{\varepsilon}}\left\langle\nabla\left(u_{\Omega_{\varepsilon}}-G\right), \nabla \psi\right\rangle(x) d x \quad \text { and } \quad J_{2,4}(\varepsilon)=\int_{\Omega_{\varepsilon}}(-f-\Delta G) \psi(x) d x .
\end{aligned}
$$

Let $B_{\varepsilon}$ stand for the function $\left\langle\nabla\left(u_{\Omega_{\varepsilon}}-G\right), \nabla \psi\right\rangle$. It follows from the argument of Remark 1 that there exists $B_{0}^{\prime} \in L^{1}(D)$ such that

$$
\frac{1}{\varepsilon}\left(B_{\varepsilon}-B_{0}\right)-B_{0}^{\prime} \longrightarrow 0 \quad \text { in } \quad L^{1}(D), \varepsilon \rightarrow 0 .
$$

Obviously, we have $\left.\frac{d}{d \varepsilon}\left(J_{2,2}(\varepsilon)\right)\right|_{\varepsilon=0^{+}}=0$ and applying Proposition 1 yields the following shape derivatives:

$$
\begin{gathered}
\left.\frac{d}{d \varepsilon}\left(J_{2,1}(\varepsilon)\right)\right|_{\varepsilon=0^{+}}=-\int_{\Omega_{2} \backslash \Omega_{1}} B_{0}^{\prime}(x) d x, \\
\left.\frac{d}{d \varepsilon}\left(J_{2,3}(\varepsilon)\right)\right|_{\varepsilon=0^{+}}=\int_{\Omega_{2} \backslash \Omega_{1}} B_{0}^{\prime}(x) d x+\int_{\Gamma_{2}} B_{0}(x) P_{\Omega_{2}^{\prime}}\left(\nu_{2}(x)\right) d \sigma, \\
\text { and }\left.\quad \frac{d}{d \varepsilon}\left(J_{2,4}(\varepsilon)\right)\right|_{\varepsilon=0^{+}}=\int_{\Gamma_{2}}(-f-\Delta G) \psi(x) P_{\Omega_{2}^{\prime}}\left(\nu_{2}(x)\right) d \sigma .
\end{gathered}
$$

Therefore,

$$
\begin{aligned}
\frac{d}{d \varepsilon} & \left.\left(J_{2}(\varepsilon)\right)\right|_{\varepsilon=0^{+}}=\int_{\Gamma_{2}}\left(B_{0}(x)-(f+\Delta G) \psi(x)\right) P_{\Omega_{2}^{\prime}}\left(\nu_{2}(x)\right) d \sigma \\
& =\int_{\Gamma_{2}}\left(\left\langle\nabla\left(u_{\Omega}-G\right), \nabla \psi\right\rangle-(f+\Delta G) \psi(x)\right) P_{\Omega_{2}^{\prime}}\left(\nu_{2}(x)\right) d \sigma,
\end{aligned}
$$

and gathering (18) and (19) gives

$$
\left.\frac{d}{d \varepsilon}\left(J_{1}(\varepsilon)+J_{2}(\varepsilon)\right)\right|_{\varepsilon=0^{+}}=\int_{\Gamma_{2}} \mathcal{B}(x) P_{\Omega_{2}^{\prime}}\left(\nu_{2}(x)\right) d \sigma
$$

where $\mathcal{B}(x)=\operatorname{div}\left(\left(u_{\Omega}(x)-\varphi_{2}(x)\right)^{2} x\right)+\left\langle\nabla\left(u_{\Omega}-G\right)(x), \nabla \psi(x)\right\rangle-(f+\Delta G)(x) \psi(x)$.

Let us show now that $J_{3}(\varepsilon)=o(\varepsilon)$, that is, $\lim _{\varepsilon \rightarrow 0^{+}} \frac{J_{3}(\varepsilon)}{\varepsilon}=0$.

We have

$$
\delta u \Delta(\delta u)=\sum_{i=1}^{n} \delta u \partial_{i}^{2} \delta u=-\sum_{i=1}^{n}\left(\partial_{i}(\delta u)\right)^{2}+\sum_{i=1}^{n} \partial_{i}\left(\delta u \partial_{i}(\delta u)\right) .
$$

It follows from (13) that $\delta u \Delta(\delta u)=0$ in $\Omega$, so that

$$
\sum_{i=1}^{n}\left(\partial_{i}(\delta u)\right)^{2}=\sum_{i=1}^{n} \partial_{i}\left(\delta u \partial_{i}(\delta u)\right) \text { in } \Omega .
$$

Applying the divergence formula yields

$$
\sum_{i=1}^{n} \int_{\Omega}\left(\partial_{i}(\delta u)\right)^{2}=\sum_{i=1}^{n} \int_{\Omega} \partial_{i}\left(\delta u \partial_{i}(\delta u)\right)(x) d x=\int_{\Gamma} \delta u \partial_{\nu}(\delta u)(x) d \sigma=\int_{\Gamma} \partial_{\nu} u_{\Omega^{\varepsilon}}(x) \delta u(x) d \sigma-\int_{\Gamma} \partial_{\nu} u_{\Omega}(x) \delta u(x) d \sigma,
$$

which can also be written as

$$
\|\nabla \delta u\|_{L^{2}(\Omega)}^{2}=\sum_{i=1}^{n} \int_{\Omega}\left(\partial_{i}(\delta u)\right)^{2}=\int_{\Gamma}\left(\partial_{\nu} u_{\Omega^{\varepsilon}}(x)-\partial_{\nu} G(x)\right) \delta u(x) d \sigma-\int_{\Gamma}\left(\partial_{\nu} u_{\Omega}(x)-\partial_{\nu} G(x)\right) \delta u(x) d \sigma
$$




$$
=\int_{\Gamma}\left(\partial_{\nu} u_{\Omega^{\varepsilon}}(x)-\partial_{\nu} G(x)\right) \delta u(x) d \sigma
$$

since $\delta u=0$ on $\Gamma_{1}$ and $\partial_{\nu_{2}} u_{\Omega}=\partial_{\nu_{2}} G$ on $\Gamma_{2}$. Moreover, it follows from the fact that $\partial_{\nu_{2}^{\varepsilon}} u_{\Omega^{\varepsilon}}=\partial_{\nu_{2}^{\varepsilon}} G$ on $\partial\left(\Omega_{2}+\varepsilon \Omega_{2}^{\prime}\right)$ and $\delta u=0$ on $\Gamma_{1}$ that $\left(\partial_{\nu^{\varepsilon}} u_{\Omega^{\varepsilon}}-\partial_{\nu^{\varepsilon}} G\right) \delta u=0$ on $\Gamma^{\varepsilon}=\partial \Omega^{\varepsilon}$, which allows us to write

$$
\|\nabla \delta u\|_{L^{2}(\Omega)}^{2}=\int_{\Gamma}\left(\partial_{\nu} u_{\Omega^{\varepsilon}}(x)-\partial_{\nu} G(x)\right) \delta u(x) d \sigma-\int_{\Gamma^{\varepsilon}}\left(\partial_{\nu^{\varepsilon}} u_{\Omega^{\varepsilon}}(x)-\partial_{\nu^{\varepsilon}} G(x)\right) \delta u(x) d \sigma,
$$

to which we apply the divergence formula to obtain

$$
\|\nabla \delta u\|_{L^{2}(\Omega)}^{2}=\int_{\Omega} \operatorname{div}\left(\nabla\left(u_{\Omega_{\varepsilon}}-G\right) \delta u\right)(x) d x-\int_{\Omega_{\varepsilon}} \operatorname{div}\left(\nabla\left(u_{\Omega_{\varepsilon}}-G\right) \delta u\right)(x) d x .
$$

Now, the fact that $-\Delta u_{\Omega_{\varepsilon}}=f$ in $\Omega_{\varepsilon}$ (hence, in $\Omega$ also) implies

$$
\operatorname{div}\left(\nabla\left(u_{\Omega^{\varepsilon}}-G\right) \delta u\right)=\left\langle\nabla\left(u_{\Omega^{\varepsilon}}-G\right), \nabla \delta u\right\rangle+\Delta\left(u_{\Omega^{\varepsilon}}-G\right) \delta u=\left\langle\nabla\left(u_{\Omega^{\varepsilon}}-G\right), \nabla \delta u\right\rangle-(f+\Delta G) \delta u,
$$

which allows us to write $\|\nabla \delta u\|_{L^{2}(\Omega)}^{2}=\Xi_{1}(\varepsilon)+\Xi_{2}(\varepsilon)+\Xi_{3}(\varepsilon)+\Xi_{4}(\varepsilon)$, where

$$
\begin{aligned}
& \Xi_{1}(\varepsilon)=\int_{\Omega}\left\langle\nabla\left(u_{\Omega^{\varepsilon}}-G\right), \nabla \delta u\right\rangle(x) d x, \quad \Xi_{2}(\varepsilon)=-\int_{\Omega}(f+\Delta G) \delta u(x) d x, \\
& \Xi_{3}(\varepsilon)=-\int_{\Omega^{\varepsilon}}\left\langle\nabla\left(u_{\Omega^{\varepsilon}}-G\right), \nabla \delta u\right\rangle(x) d x \quad \text { and } \quad \Xi_{4}(\varepsilon)=\int_{\Omega^{\varepsilon}}(f+\Delta G) \delta u(x) d x .
\end{aligned}
$$

Denote by $W_{\varepsilon}$ the function defined by $W_{\varepsilon}=\left\langle\nabla\left(u_{\Omega_{\varepsilon}}-G\right), \nabla \delta u\right\rangle$ and by $H_{\varepsilon}$ the function defined by $H_{\varepsilon}=(f+\Delta G) \delta u$, with $W_{0}=0$ and $H_{0}=0$. Once more, the argument of Remark 1 implies that there exist $W_{0}^{\prime} \in L^{1}(D)$ and $H_{0}^{\prime} \in L^{1}(D)$ such that, when $\varepsilon \rightarrow 0$,

$$
\frac{1}{\varepsilon}\left(W_{\varepsilon}-W_{0}\right)-W_{0}^{\prime} \longrightarrow 0 \text { and } \frac{1}{\varepsilon}\left(H_{\varepsilon}-H_{0}\right)-H_{0}^{\prime} \longrightarrow 0,
$$

in $L^{1}(D)$. This allows us to apply Proposition 1 to obtain

$$
\begin{gathered}
\left.\frac{d}{d \varepsilon} \Xi_{1}(\varepsilon)\right|_{\varepsilon=0^{+}}=-\int_{\Omega_{2} \backslash \Omega_{1}} W_{0}^{\prime}(x) d x,\left.\quad \frac{d}{d \varepsilon} \Xi_{2}(\varepsilon)\right|_{\varepsilon=0^{+}}=-\int_{\Omega_{2} \backslash \Omega_{1}} H_{0}^{\prime}(x) d x \\
\left.\frac{d}{d \varepsilon} \Xi_{3}(\varepsilon)\right|_{\varepsilon=0^{+}}=\int_{\Omega_{2} \backslash \Omega_{1}} W_{0}^{\prime}(x) d x+\int_{\Gamma_{2}} W_{0}(x) P_{\Omega_{2}^{\prime}}\left(\nu_{2}(x)\right) d \sigma=\int_{\Omega_{2} \backslash \Omega_{1}} W_{0}^{\prime}(x) d x
\end{gathered}
$$

and

$$
\left.\frac{d}{d \varepsilon} \Xi_{4}(\varepsilon)\right|_{\varepsilon=0^{+}}=\int_{\Omega_{2} \backslash \Omega_{1}} H_{0}^{\prime}(x) d x+\int_{\Gamma_{2}} H_{0}(x) P_{\Omega_{2}^{\prime}}\left(\nu_{2}(x)\right) d \sigma=\int_{\Omega_{2} \backslash \Omega_{1}} H_{0}^{\prime}(x) d x .
$$

Consequently, $\left.\frac{d}{d \varepsilon}\|\nabla \delta u\|_{L^{2}(\Omega)}^{2}\right|_{\varepsilon=0^{+}}=0$, which implies that $\|\nabla \delta u\|_{L^{2}(\Omega)}^{2}=o(\varepsilon)$. It remains to apply the Poincaré inequality: there exists a constant $C_{\Omega}$ such that

$$
\|\delta u\|_{L^{2}(\Omega)}^{2} \leq C_{\Omega}\|\nabla \delta u\|_{L^{2}(\Omega)}^{2},
$$

and

$$
\left|2 \int_{\Omega} \delta u\left\langle\nabla(\delta u), I_{d}\right\rangle\right| \leq C|| \delta u\left\|_{L^{2}(\Omega)}\right\| \nabla \delta u\left\|_{L^{2}(\Omega)} \leq C C_{\Omega}\right\| \nabla \delta u \|_{L^{2}(\Omega)}^{2},
$$

with some positive constant $C$. These two inequalities imply of course that

$$
J_{3}(\varepsilon)=n\|\delta u\|_{L^{2}(\Omega)}^{2}+2 \int_{\Omega} \delta u(x)\langle\nabla(\delta u)(x), x\rangle d x=o(\varepsilon) .
$$

Thus, recalling (20), we have proved that

$$
\triangle \mathcal{J}=J_{1}(\varepsilon)+J_{2}(\varepsilon)+J_{3}(\varepsilon)=\varepsilon \int_{\Gamma_{2}} \mathcal{B}(x) P_{\Omega_{2}^{\prime}}\left(\nu_{2}(x)\right) d \sigma+o(\varepsilon),
$$

and this achieves the proof of the theorem. 


\section{$5 \quad$ Identification process for optimal domain}

The numerical optimization process used for the estimation of the unknown free boundary $\Gamma_{2}$ of $\Omega$ is based on the gradient method presented in the following.

\subsection{Gradient Method}

Using the gradient of $\mathcal{J}$ determined by equation (5), the computation of the optimal shape is summarized in the following algorithm.

1. Choose $\left.\Omega_{0}=\Omega_{0,2} \backslash \Omega_{1} \in \mathcal{O}, \rho \in\right] 0,1[$ and a precision $E P S D$.

2. Solve the state problem

$$
\left\{\begin{array}{lll}
\Delta u=f & \text { in } & \Omega_{0} \\
u=\varphi_{1} & \text { on } & \Gamma_{1} \\
\partial_{\nu_{2}} u=\partial_{\nu_{2}} G & \text { on } & \Gamma_{0,2} .
\end{array}\right.
$$

3. Solve the adjoint state problem

$$
\left\{\begin{array}{lll}
\Delta \psi=0 & \text { in } & \Omega_{0}, \\
\psi=0 & \text { on } & \Gamma_{1}, \\
\partial_{\nu_{2}} \psi=-2\left(u-\varphi_{2}\right)\left\langle\nu_{2}(x), x\right\rangle & \text { on } & \Gamma_{0,2} .
\end{array}\right.
$$

4. Compute $\mathcal{B}$ by the relation

$$
\left.\mathcal{B}(x)=\operatorname{div}\left(\left(u-\varphi_{2}\right)^{2} x\right)+(\nabla(u-G), \nabla \psi)-(f+\Delta G) \psi\right) .
$$

5. Compute $P_{0}=P_{\Omega_{0,2}}$

6. Compute $\widehat{P}$ the solution of

$$
\arg \min _{P \in \mathcal{P}} j(P)
$$

where

$$
j(P)=\int_{\Gamma_{0,2}} B(x)\left(P-P_{0}\right)(\nu(x)) d s
$$

and

$$
\mathcal{P}=\left\{\Phi \in C\left(\mathbb{R}^{2}\right) / \Phi \text { is convex and } P_{\Omega_{1}} \leq \Phi \leq P_{D}\right\} .
$$

7. At step $k$

$$
\text { if }\left\|u^{k}-\varphi_{2}\right\|_{L^{2}\left(\Gamma_{k, 2}\right)} \leq E P S D \quad \text { Go to } \mathbf{1 0} .
$$

where $u^{k}$ is the solution of the state equation in $\Omega_{k}$ (the domain at step $k$ ) and $\Gamma_{k, 2}$ is the outside boundary of $\Omega_{k}$.

8. Compute

$$
P_{k+1}=(1-\rho) P_{k}+\rho \widehat{P}_{k}
$$

9. Compute the domain $\bar{\Omega}_{k+1}$ associated to $P_{k+1}$ by

$$
\bar{\Omega}_{k+1}=\partial P_{k+1}(0)=\left\{l \in \mathbb{R}^{2} / P_{k+1}(x) \geq(l, x), \forall x \in \mathbb{R}^{2}\right\} \quad \text { Go to } 2 .
$$

10. End 


\subsection{Discretization of the problem}

In this section, we approach the optimal shape design problem (5) using the boundary element method. For this, the state problem (24) and the adjoint state problem (25) can be written in terms of boundary integral equation [10] by the equation

$$
c(\xi) u(\xi)=\int_{\partial \Omega}\left\{u(y) \partial_{\nu_{y}} \ln |\xi-y|\right\} d s_{y}-\partial_{\nu_{y}} u(y) \ln |\xi-y| d s_{y}
$$

where $c(\xi)$, called the free term, is 0 if $\xi \notin \Omega \cup \partial \Omega, 1$ if $\xi \in \Omega$ and $\theta / 2 \pi$ if $\xi \in \partial \Omega$, where $\theta$ is the interior angle between the left and right tangents to the boundary at the point $\xi ; u^{*}(y ; \xi)=(1 / 2 \pi) \ln (1 / r)$ is the fundamental solution for the Laplace equation; $r=|y-\xi|$ is the distance between the collocation point $\xi$ and the integration or observation point $y$; and $q^{*}=-(1 / 2 \pi) \partial u^{*} / \partial \nu$ is the flux associated to the potential $u^{*}(y ; \xi)$.

The subdivision of the boundary can be done using different types of elements. In the present investigation, constant elements are considered. Assuming that over each boundary element $\partial \Omega_{i}$ of $\partial \Omega$ the functions $u$ and $q=\frac{\partial u}{\partial \nu}$ are constant and take their values $u_{i}$ and $q_{i}$ at the centroid $\xi_{i}$, the resulted discretized boundary integral equation at node $k$ has the following form

$$
\sum_{l=1}^{N E} H_{k l} u_{l}=\sum_{l=1}^{N E} G_{k l} q_{l}
$$

where $N E$ is the number of boundary elements $\partial \Omega_{i}$ such that $\partial \Omega=\cup_{i=1}^{N E} \partial \Omega_{i}$. The components of the coefficient matrices $H$ and $G$ are given by

$$
\begin{aligned}
H_{k l} & =\int_{\partial \Omega_{l}} q_{k}^{*} \text { when } k \neq l, \\
H_{k l} & =\int_{\partial \Omega_{l}} q_{k}^{*}+\frac{1}{2} \text { when } k=l, \\
G_{k l} & =\int_{\partial \Omega_{l}} u_{k}^{*} .
\end{aligned}
$$

A four-point Gaussian quadrature rule is employed for numerical integration of the components of the coefficient matrices $H$ and $G$. However, for calculating the diagonal components, special care must be taken. The diagonal terms $H_{k k}$ vanish due to the orthogonality of the element coordinate and the normal. The terms $G_{k k}$ can be calculated analytically and it is given by the following equation

$$
G_{k k}=\frac{L_{e}}{2 \pi}\left[\ln \left(\frac{2}{L_{e}}\right)+1\right]
$$

where $L_{e}$ is the element length. The values of the off-diagonal coefficients of $H$ and $G$ can be written as [10]

$$
\begin{aligned}
& H_{k l}=\sum_{i=1}^{4}-\left(\frac{1}{d_{s}^{2}}\right) L w_{s} \frac{\sqrt{\left(R_{1}-R_{2}\right)^{2}+\left(S_{1}-S_{2}\right)^{2}}}{2} \\
& G_{k l}=\sum_{i=1}^{4} \ln \left(\frac{1}{d_{s}}\right) w_{s} \frac{\sqrt{\left(R_{1}-R_{2}\right)^{2}+\left(S_{1}-S_{2}\right)^{2}}}{2}
\end{aligned}
$$

where $R_{1}, R_{2}, S_{1}$ and $S_{2}$ are the coordinates of the extreme mounts of each element, $w_{s}$ are the weighting for each point, $L$ is the distance from the collocation point to the line element tangent to the element and $d$ is the distance from collocation point to the Gauss integration points on the boundary element. We note that the system (28) can easily be rearranged as a system of order $n$ and written in a generic form

$$
A x=b
$$


Its right-hand side is obtained by multiplication of the appropriate columns of the influence matrices $H$ and $G$ by values known from boundary conditions. Components of the vector of the unknowns $x$ as well as components of the main matrix $A$ depend on the boundary condition at the considered node. We obtain

$$
\begin{gathered}
A_{i j}= \begin{cases}-G_{i j} & \text { for Dirichlet's boundary condition } \\
H_{i j} & \text { for Neumann's boundary condition }\end{cases} \\
x^{j}= \begin{cases}q_{j} & \text { for Dirichlet's boundary condition } \\
u_{j} & \text { for Neumann's boundary condition }\end{cases}
\end{gathered}
$$

Let now $m$ be the number of the boundary elements located at the free boundary $\Gamma_{2}$, such that $m<n$ and $n-m$ is the number of boundary elements located at the inside fixed boundary $\Gamma_{1}$ of $\Omega$. Let $Y=\left(y_{i}\right)_{i=0}^{m}$ be a partition of $\Gamma_{2}$, where $y_{i}=\left(y_{i}^{(1)}, y_{i}^{(2)}\right)$, we denote by $\xi=\left(\xi_{i}\right)_{i=0}^{m}$ a partition of $\Gamma_{2}$, such that $\xi_{i}=\left(\xi_{i}^{(1)}, \xi_{i}^{(2)}\right)$ is the centroid of the boundary elements $\left[y_{i}, y_{i+1}\right]$.

The discrete gradient of the cost function can be read as follows

$$
j(P)=\sum_{i=0}^{m} h_{i} B_{i}\left(P_{i}-P_{\Omega}\left(\nu\left(\xi_{i}\right)\right)\right)
$$

where $h_{i}=\left\|y_{i+1}-y_{i}\right\| ; P=\left(P_{i}=P\left(\nu\left(\xi_{i}\right)\right)_{i=0}^{m} ; \nu\left(\xi_{i}\right)\right.$ is the outward unit normal vector to the boundary elements $y_{i} y_{i+1}$ and $B_{i}=B\left(\xi_{i}^{(1)}, \xi_{i}^{(2)}\right)$ is given by the relation

$$
\begin{aligned}
B_{i} & =2\left(u_{i}-\varphi_{2}\left(\xi_{i}\right)\right)\left(\nabla u^{h}\left(\xi_{i}\right)-\nabla \varphi_{2}\left(\xi_{i}\right), \xi_{i}\right)+2\left(u_{i}-\varphi_{2}\left(\xi_{i}\right)\right)^{2} \\
& \left.+\left(\nabla\left(u^{h}\left(\xi_{i}\right)-G^{h}\left(\xi_{i}\right)\right), \nabla \psi^{h}\left(\xi_{i}\right)\right)+\left(f\left(\xi_{i}\right)-\Delta G^{h}\left(\xi_{i}\right)\right) \psi_{i}\right)
\end{aligned}
$$

where $u^{h}$ is the approximate solution of the state equation, such that $\left(u^{h}(\xi)=u_{i}\right)_{i=0}^{n}$ is the solution of a linear system of type (35), $\psi^{h}$ is the approximate solution of the adjoint state equation, such that $\left(\psi^{h}(\xi)=\psi_{i}\right)_{i=0}^{n}$ is the solution of a linear system of type (35) and $G^{h}$ is a approximation of $G$. The discrete space of the admissible support function can be read

$$
\begin{gathered}
\mathcal{P}=\left\{P=\left(P_{0}, \ldots, P_{m}\right) \in \mathbb{R}^{m+1} / P_{\Omega_{1}}\left(\nu\left(\xi_{i}\right)\right) \leq P_{i} \leq P_{D}\left(\nu\left(\xi_{i}\right)\right) i=1, \ldots, m-1, P_{0} \leq \lambda_{0} P_{m}+\left(1-\lambda_{0}\right) P_{1},\right. \\
\left.P_{i} \leq \lambda_{i} P_{i-1}+\left(1-\lambda_{i}\right) P_{i+1} \quad i=1, \ldots, m-1 \text { and } P_{m} \leq \lambda_{m} P_{m-1}+\left(1-\lambda_{m}\right) P_{0}\right\}
\end{gathered}
$$

where

and

$$
\lambda_{i}=\frac{\left\|\nu\left(\xi_{i-1}\right)-\nu\left(\xi_{i}\right)\right\|}{\left\|\nu\left(\xi_{i}\right)-\nu\left(\xi_{i+1}\right)\right\|} \quad \text { for } i=1, \ldots, m-1
$$

$$
\lambda_{0}=\frac{\left\|\nu\left(\xi_{m}\right)-\nu\left(\xi_{0}\right)\right\|}{\left\|\nu\left(\xi_{0}\right)-\nu\left(\xi_{1}\right)\right\|} \quad \lambda_{m}=\frac{\left\|\nu\left(\xi_{m-1}\right)-\nu\left(\xi_{m}\right)\right\|}{\left\|\nu\left(\xi_{m}\right)-\nu\left(\xi_{0}\right)\right\|} .
$$

The discrete domain associated to $\bar{\Omega}_{0}=\partial \bar{P}(0)=\left\{l \in \mathbb{R}^{2} / \bar{P}(x) \geq(l, x), \forall x \in \mathbb{R}^{2}\right\}$ is given as solution of the following relations [24]

$$
\nu^{(1)}\left(\xi_{i}\right) l_{i}^{(1)}+\nu^{(2)}\left(\xi_{i}\right) l_{i}^{(2)}=\bar{P}_{i} \quad \text { for } i=0, \ldots, m
$$

To compute $l_{i}=\left(l_{i}^{(1)}, l_{i}^{(2)}\right)$, we suppose that $l_{i}-\xi_{i}$ is collinear to $\nu\left(\xi_{i}\right)$ i.e.

$$
\nu^{(1)}\left(\xi_{i}\right) l_{i}^{(2)}-\nu^{(2)}\left(\xi_{i}\right) l_{i}^{(1)}=\nu^{(1)}\left(\xi_{i}\right) \xi_{i}^{(2)}-\nu^{(2)}\left(\xi_{i}\right) \xi_{i}^{(1)} \text { for } i=0, \ldots, m
$$

\section{Numerical results}

To illustrate the ability of the present algorithm in estimating the optimal shape and location of the unknown boundary $\Gamma_{2}$, we consider in the following two the specific models: the analysis one and the design one. 
In the analysis model, the exact boundary $\Gamma_{2}$ and the exact solution $u$ of the free boundary problem (5) are known. By using the exact value of $u$ on $\Gamma_{1}$ and $\Gamma_{2}$, and arbitrary initial guesses of the boundary, one is asked to reconstruct the exact boundary by the gradient method combined with our proposed method used for the computation of the gradient.

The design model is devoted to the investigation of some numerical experiment based on the approaches proposed in the previous works to explore free boundary problems of Bernoulli type.

\subsection{Analysis model}

The main objective of the analysis model is to show the validity of the present design algorithm in estimating the unknown boundary and to verify the accuracy of our method in the computation of the shape derivative of the cost function. For this reason the following numerical simulation is performed. For the first example, we consider the exact solution of (2) given by $u(x, y)=x^{2}-y^{2}$ and the exact domain given by considering $\Omega=\Omega_{2} \backslash \Omega_{1}$, where

$$
\begin{gathered}
\Omega_{2}=\left\{(x, y) / x^{2}+y^{2}<(1.5)^{2}\right\} \\
\Omega_{1}=\left\{(x, y) / x^{2}+y^{2} \leq(0.75)^{2}\right\}
\end{gathered}
$$

In all the test cases considered for this analysis model, the initial guesses of the domain $\Omega_{2}$ is taken as a ellipse with axes of length 3 in $x$ and 2 in $y$ and of center $(0,0)$. The number of boundary elements used for the discretization of the free boundary and the fixed boundary were taken to be respectively $m=40$ and $n-m=30(n=70)$. The coefficient of deformation and the precision are respectively taken $\rho=0.01$ and $E P S D=10^{-3}$. The optimal boundary is reached after 181 iterations. In figure 6.1, we plot the fixed and initial domain, and the exact and the optimal solution. We note that our approach give a good solution. the variation of the cost function with respect to the number of iterations is presented in figure 6.1. In figure 6.1, we plot the initial boundary, the free boundaries at $10,40,80,120$ and 150 iterations.

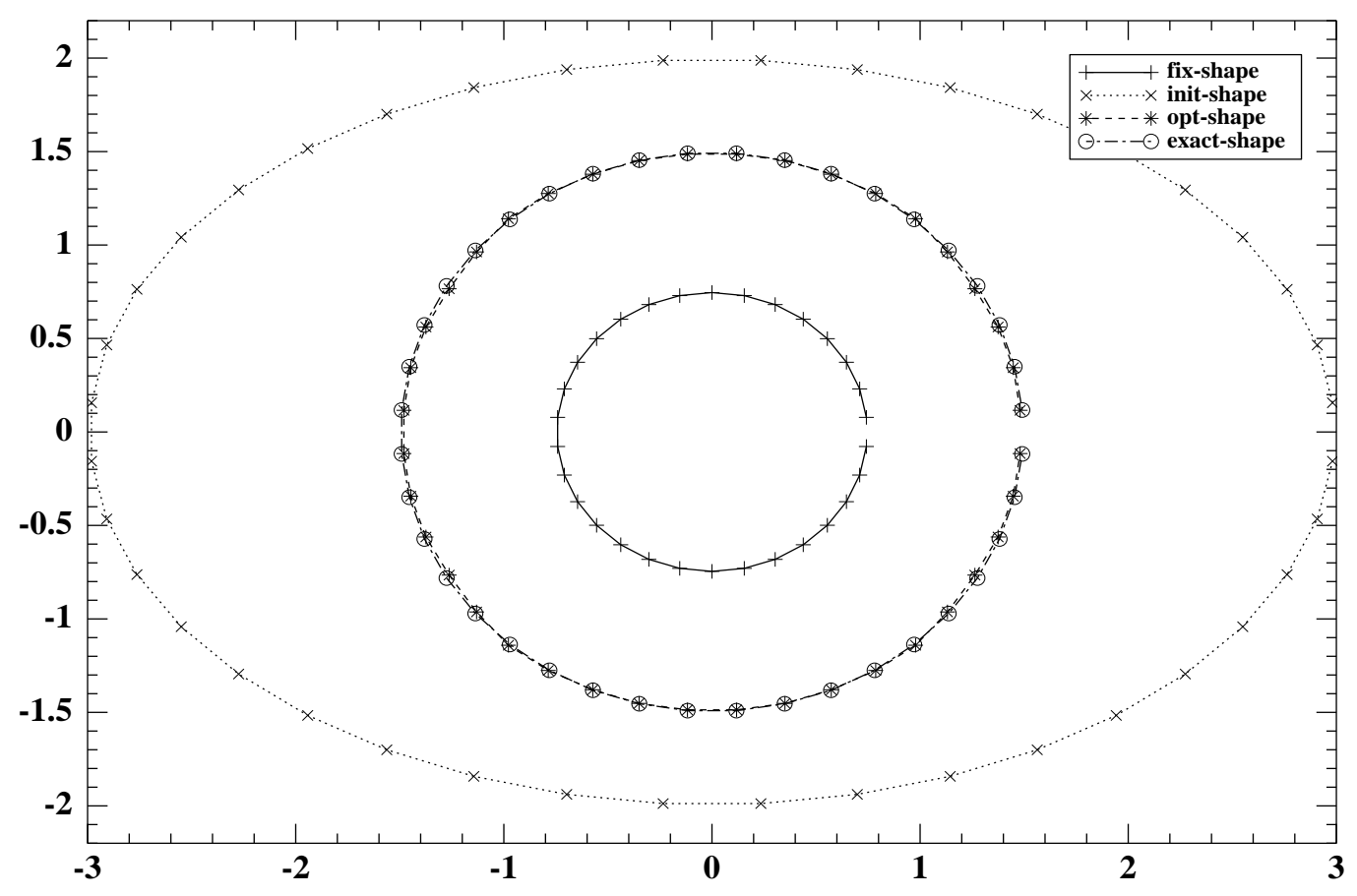

Figure 2: The optimal and the exact solutions 


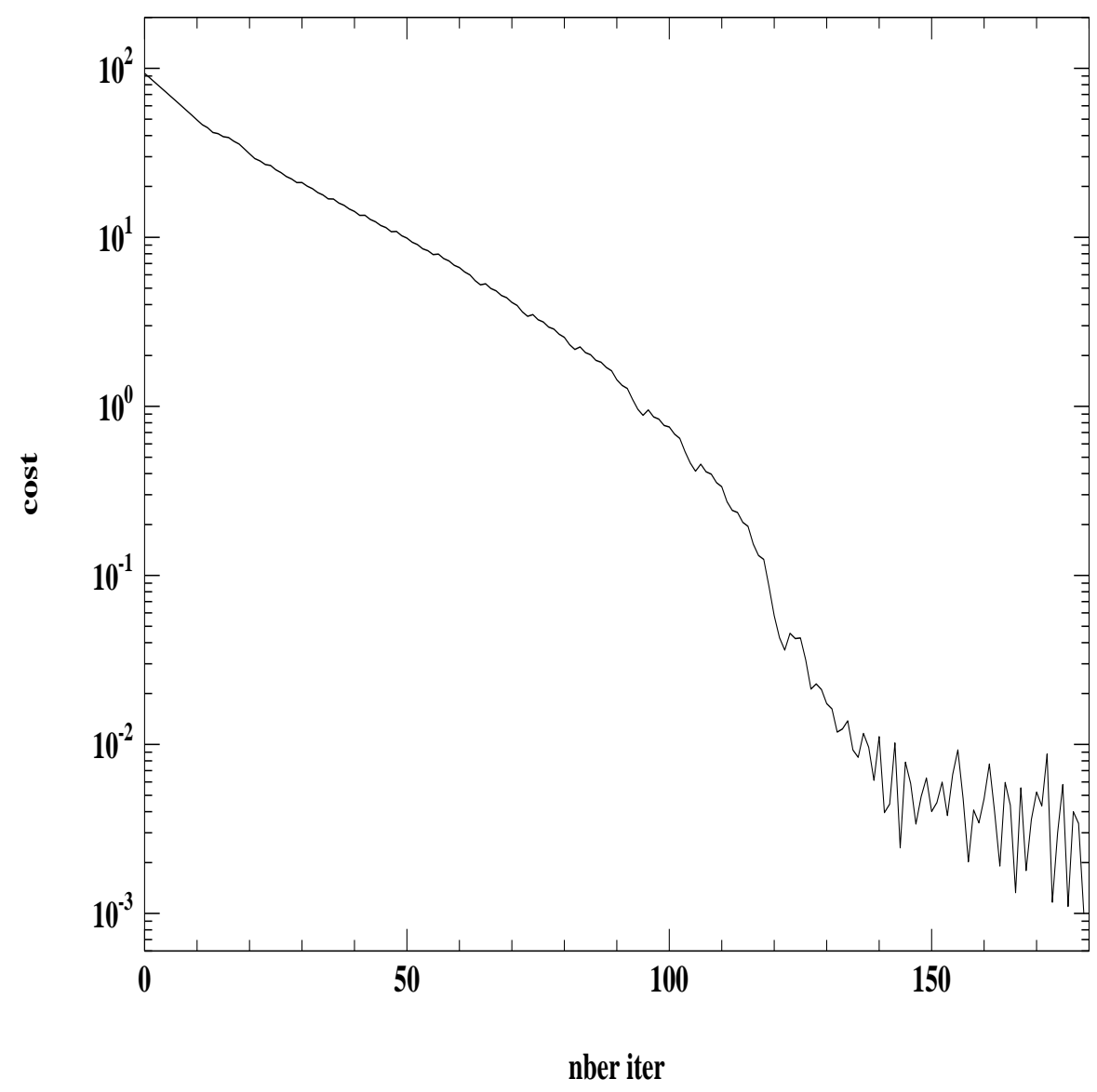

Figure 3: Variation of the cost with respect to the number of iterations

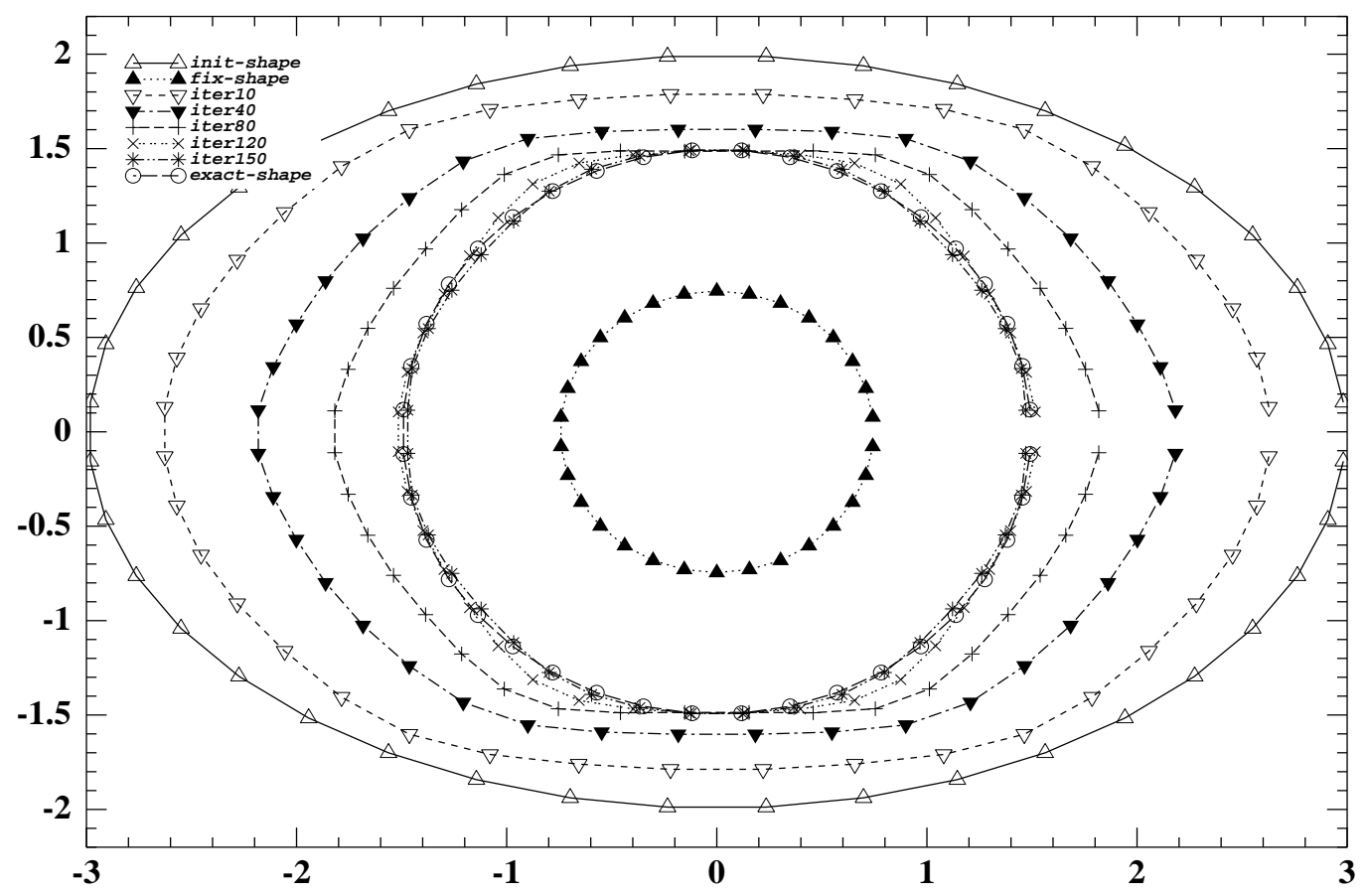

Figure 4: the free boundaries at different number of iterations. 
Now we keep the same solution $u=x^{2}-y^{2}$, but the exact domain is defined by the ellipse with axes of length 1.5 in $x$ and 1 in $y$ and of center $(0,0)$. For the same number of boundary elements and for the coefficient of deformation and the precision respectively taken $\rho=0.0001$ and $E P S D=10^{-3}$ the optimal boundary is reached after 111 iterations. The fixed and the initial domain, and the exact and the optimal solution are presented in figure 6.1. In figure 6.1, we plot the variation of the cost function with respect to the number of iterations.

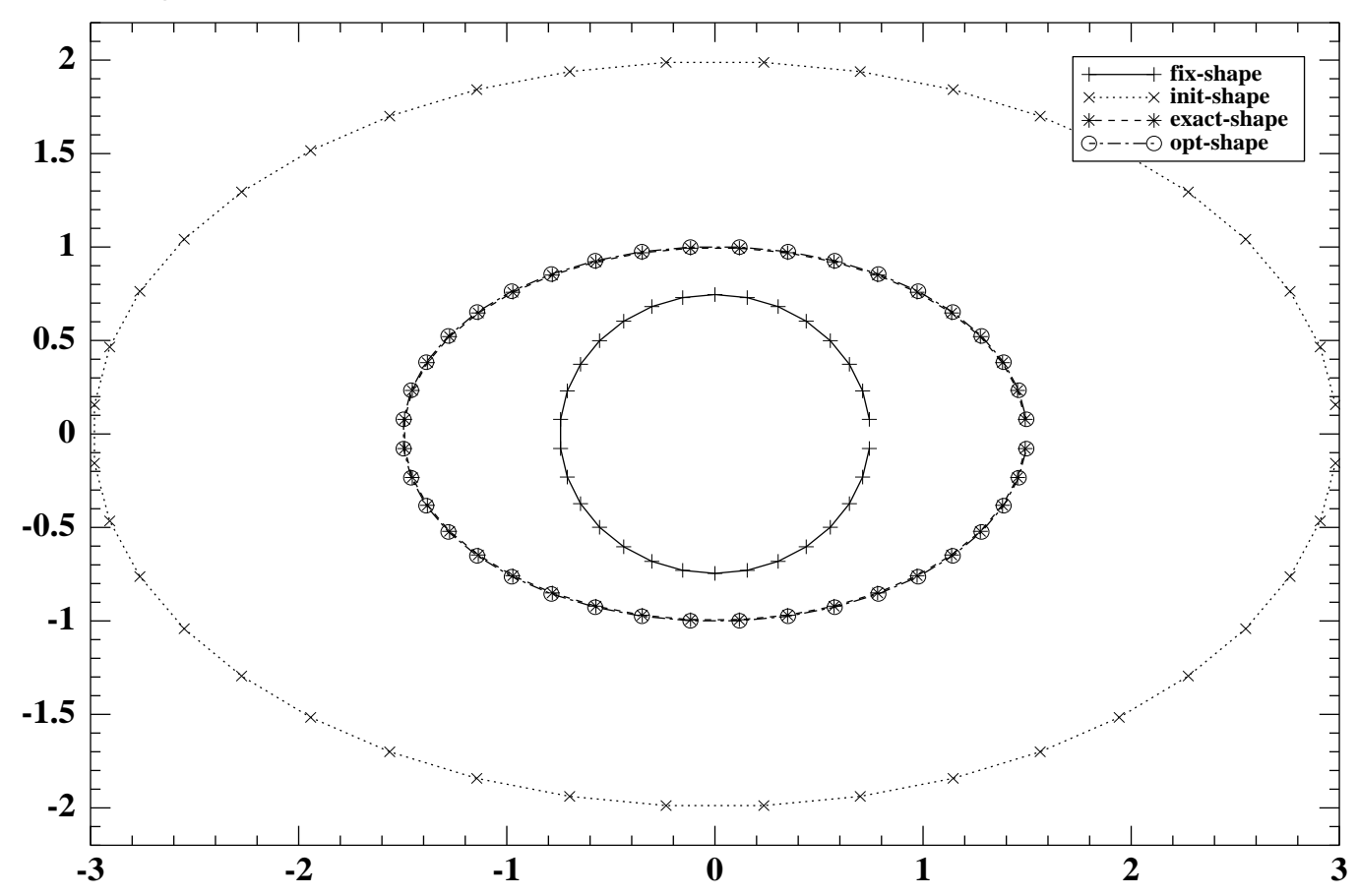

Figure 5: The optimal and the exact solutions. 


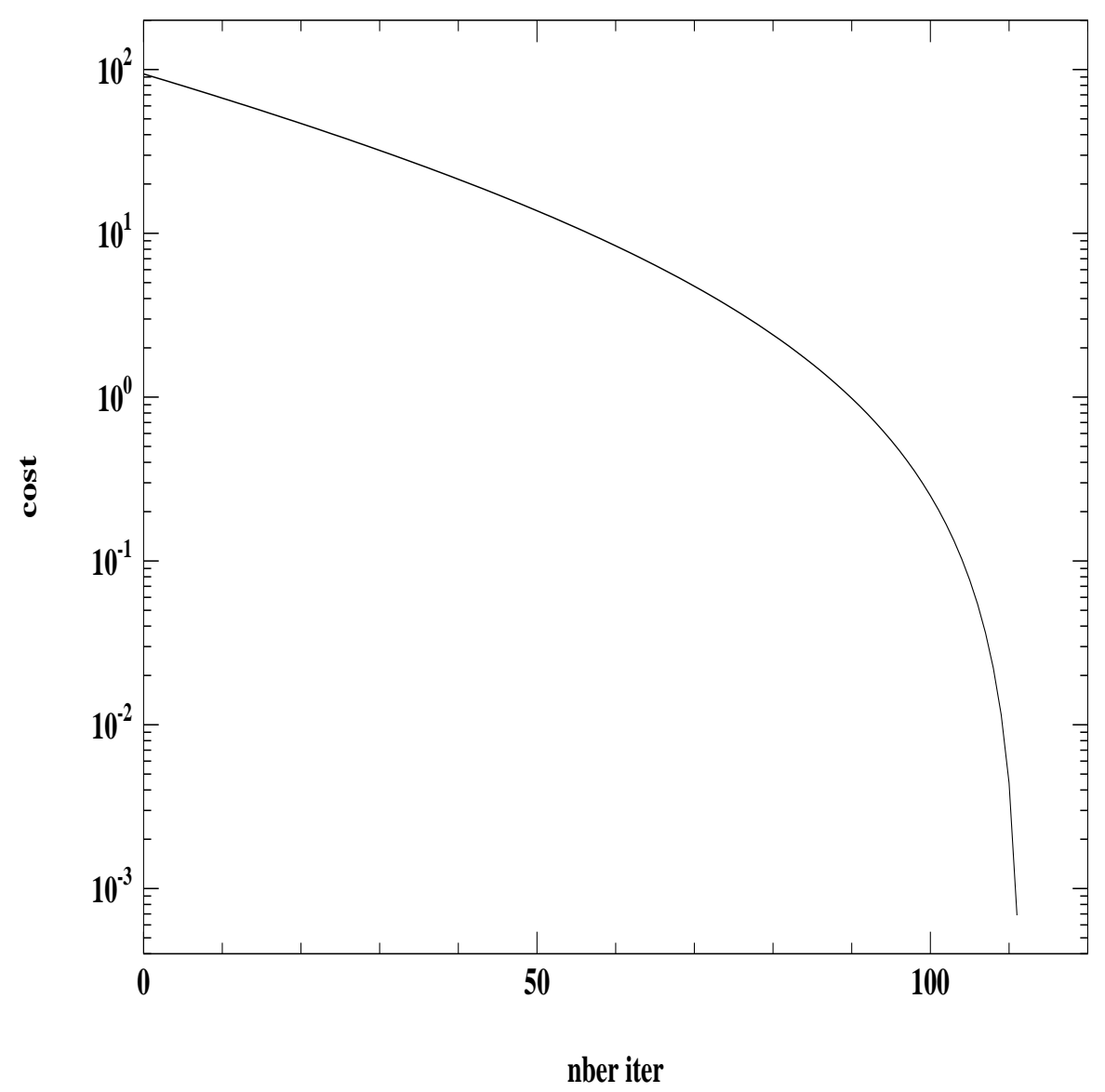

Figure 6: Variation of the cost with respect to the number of iterations.

\subsection{Design model}

In this section, we present some numerical results for the exterior free boundary problems using the approach presented in the previous section. The exterior Bernoulli free boundary reads as follows. Find outside boundary $\Gamma_{2}$ and function $u=u(x)$ solution of the problem

$$
\left\{\begin{array}{lll}
-\Delta u=0 & \text { in } & \Omega, \\
u=0 & \text { on } & \Gamma_{1}, \\
u=1 & \text { on } & \Gamma_{2}, \\
\frac{\partial u}{\partial \nu}=C & \text { on } & \Gamma_{2}
\end{array}\right.
$$

where $C$ is a negative constant.

The first numerical example is given in order to confirm the fundamental observation (see [16]), that is for a fixed domain $\Omega_{1}$ which is the ball $B_{0}^{r}$ of center $(0,0)$ and radius $r$ a solution is a ball $B_{0}^{R}$ of center $(0,0)$ and radius $R$, such that $R>r$; and moreover $R$ increases when $C$ increases. In all the test cases considered for this design model, the initial guesses of the domain is taken as a circle of radius 3 . and of center $(0,0)$. In the first example, the fixed domain is chosen as circle of radius 0.75 and of center $(0,0)$, and the number of boundary elements used for the discretization of the free and fixed boundaries are taken the same as in the above examples. The coefficient of deformation and the precision are respectively taken $\rho=0.01$ and $E P S D=10^{-5}$. The optimal domains for different values of $C$ are presented in figure 6.2. The values of the cost functions for different values of $C$ are given in table 6.2. 


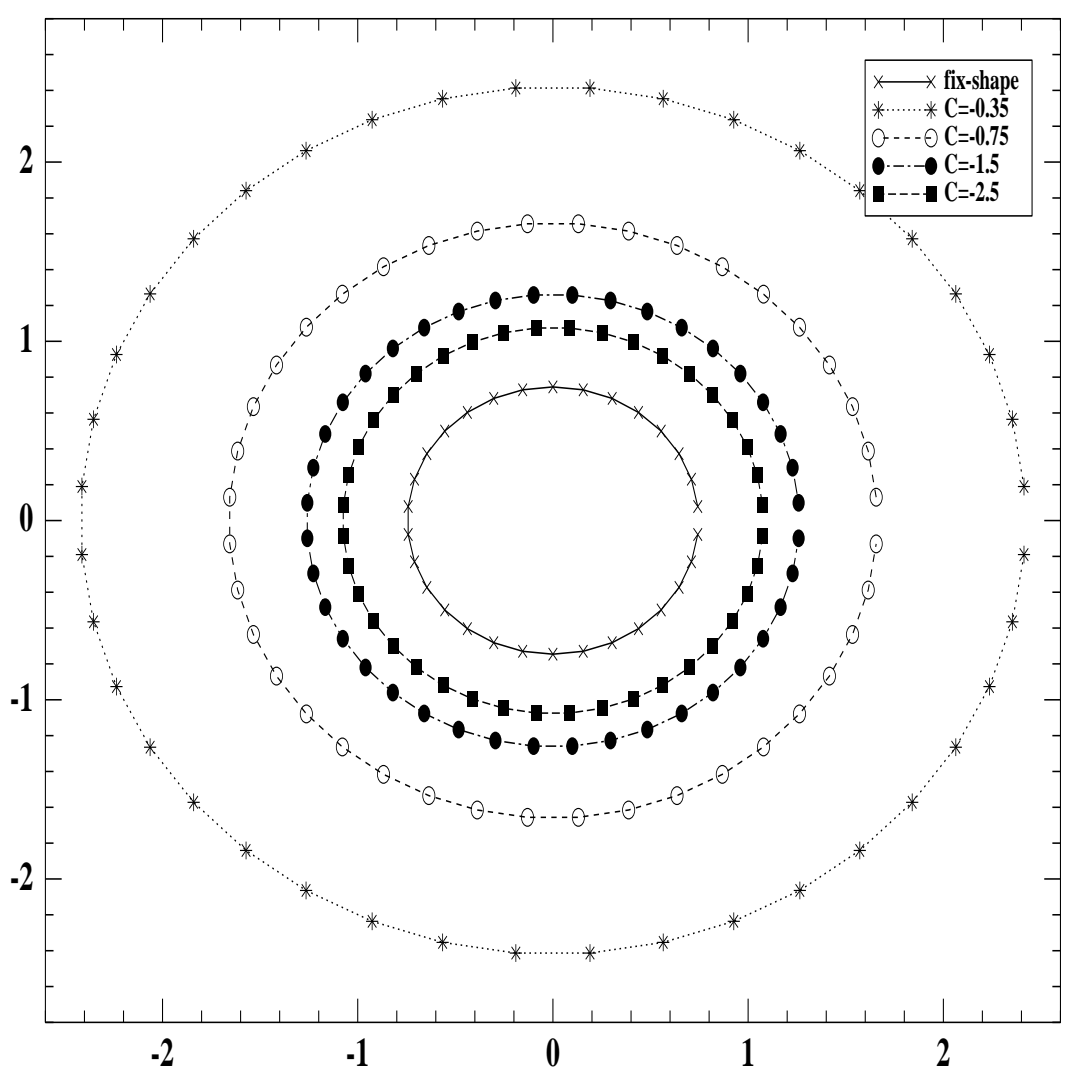

Figure 7: The optimal domains for different values of $C$.

\begin{tabular}{ccccc}
\hline $\mathrm{C}$ & -0.35 & -0.75 & -1.5 & -2.5 \\
\hline Cost & $1.5510^{-6}$ & $8.5510^{-8}$ & $4.310^{-8}$ & $4.3410^{-6}$ \\
\hline
\end{tabular}

Table 1: Cost functions for different values of $C$.

Now for the second example, we keep the same data, but the fixed domain $\Omega_{1}$ is taken as a polygon. The number of boundary elements used for the discretization of the free boundary and the fixed boundary were taken to be respectively $m=40$ and $n-m=40(n=80)$. The fixed domain and the optimal domains for different values of $C$ are presented in figure 6.2. We observe that the optimal shapes take the same profile to those obtained in $[18,21]$. 


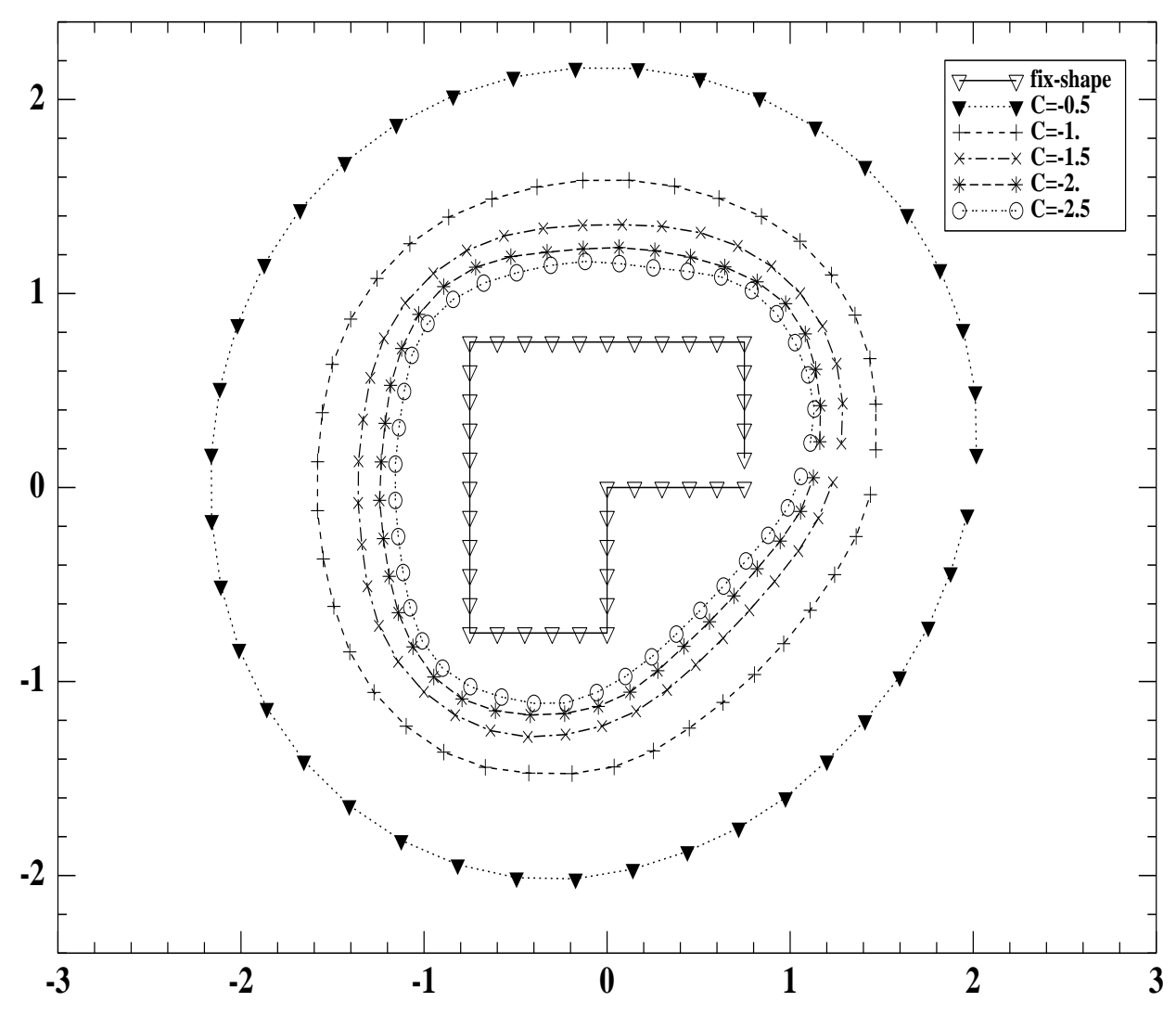

Figure 8: The optimal domains for different values of $C$.

\section{Conclusion}

In this work, a shape optimization formulation is considered for the approximation of the free boundary Bernoulli problem. We show the existence of the shape derivative of the cost functional and establish its expression via support functions, in order to avoid the tedious use of the shape derivative method based on the vector fields. Then the gradient method combined with the boundary element method are performed for the discretization of the problem. Finally we give some numerical results showing the ability and effectiveness of the proposed approach.

\section{References}

[1] Acker, A. and Meyer, R., A free boundary problem for the p-Laplacian: uniqueness, convexity, and successive approximation of solutions. Electronic Journal of Differential Equations. 8 (1995) 1-20.

[2] Allaire, G., Conception optimale de structures. Mathématiques et Applications. Berlin: Springer, 58, 2007.

[3] Alt, H., W. and Caffarelli, L., A. Existence and regularity for a minimum problem with free boundary. J. Reine Angew. Math. 325 (1981) 105144.

[4] Antunes, P., B. Bogosel, Parametric Shape Optimization using the Support Function. Available at https://arxiv.org/pdf/1809.00254.pdf (2018). 
[5] Bouchon, F., Clain, S. and Touzani, R., Numerical solution of the free boundary Bernoulli problem using a level set formulation. Computer methods in applied mechanics and engineering. 194. 36-38 (2005) 3934-3948.

[6] Boulkhemair, A. On a Shape Derivative Formula in the Brunn-Minkowski Theory. SIAM Journal on Control and Optimization. 55. 1 (2017) 156-171.

[7] Boulkhemair, A. and Chakib, A., On a shape derivative formula with respect to convex domains. Journal of Convex Analysis. 21. 1 (2014) 67-87.

[8] Boulkhemair, A. and Chakib, A., Erratum: On a shape derivative formula with respect to convex domains. $J$. Convex Anal. 22 (2015) 901-903.

[9] Boulkhemair, A., Chakib, A. and Nachaoui, A., A shape optimization appoach for a class of free boundary problems of Bernoulli type. Applications of Mathematics. 58. 2 (2013) 205-221.

[10] Brebbia, C. A., and Dominguez, J., Boundary elements: an introductory course. Comp. Mech. Pub. McGrawHill Book Company, 1992.

[11] Cardaliaguet, P. and Tahraoui, R., Some uniqueness results for Bernoulli interior free boundary problems in convex domains. Electronic Journal of Differential Equations (EJDE). 102. 2 (2002).

[12] Céa, J., Approximation variationnelle des problèmes aux limites. Ann. Inst. Fourier (Grenoble). 14. fasc. 2 (1964) 345-444.

[13] Delfour, M. C. and Zolésio, J., P., Shapes and geometries: metrics, analysis, differential calculus, and optimization. Siam, 22, 2011.

[14] Eppler, K. and Harbrecht, H., Tracking Neumann Data for Stationary Free Boundary Problems.SIAM Journal on Control and Optimization. 48. 5 (2009) 2901-2916.

[15] Eppler, K. and Harbrecht, H., Tracking Dirichlet Data in L ${ }^{2}$ is an Ill-Posed Problem. Journal of optimization theory and applications 145. 1 (2010) 17-35.

[16] Flucher, M., and Rumpf, M. Bernoulli's free-boundary problem, qualitative theory and numerical approximation. Journal fur die Reine und angewandte Mathematik. 486 (1997) 165-204.

[17] Haslinger, J., Kozubek, T., Kunisch, K., Peichl, G., An embedding domain approach for aclass of 2-d shape optimization problems: Mathematical analysis. J. Math. Anal. Appl. 290 (2004) 665685.

[18] Haslinger, J., Kozubek, T., Kunisch, K., Peichl, G., Shape optimization and fictitious domain approach for solving free-boundary value problems of Bernoulli type, Comput. Optim. Appl. 26 (2003) 231-251.

[19] Henrot, A., and Pierre, M., Variation et optimisation de formes : une analyse géométrique. Springer Science \& Business Media, 48, 2006.

[20] Hörmander, L., Notions of convexity, Progress in Mathematics, 127. Birkhäuser Boston, Inc., Boston, MA, 1994.

[21] Ito, K., Kunisch, K. and G., H., Peichl., Variational approach to shape derivatives for a class of Bernoulli problems. Journal of Mathematical Analysis and Applications. 314.1 (2006) 126-14

[22] Kinderlehrer, D., and Nirenberg, L., Regularity in free boundary problems. Annali della Scuola Normale Superiore di Pisa-Classe di Scienze. 4. 2 (1977) 373-391.

[23] Kuster, C. M., Pierre A., G. and Touzani, R., Fast numerical methods for Bernoulli free boundary problems. SIAM journal on scientific computing. 29. 2 (2007) 622-634. 
[24] Niftiyev, A.A.; Gasimov Y.S. Control by boundaries and eigenvalue problems with variable domains, Publishing House of Baku State University, (in Russian), 2004.

[25] Rabago, Julius Fergy, T. and Bacani, Jerico, B., Shape optimization approach for solving the Bernoulli problem by tracking the Neumann data: A Lagrangian formulation, Communications on Pure E Applied Analysis, 17. 6 (2018) 2683-2702.

[26] Sokolowski, J., and Zolesio, J., P., Introduction to Shape Optimization. Springer, Berlin, Heidelberg, 5-12, 1992.

[27] Schneider, R., Convex bodies: the BrunnMinkowski theory. Cambridge university press, 151, 2014.

[28] Toivanen, J. I., Haslinger, J. , and Mkinen, R., A., E., Shape optimization of systems governed by Bernoulli free boundary problems. Computer Methods in Applied Mechanics and Engineering. 197. 45-48 (2008) 3803-3815. 